\title{
Toward Joint Radar, Communication, Computation, Localization, and Sensing in loT
}

This paper was downloaded from TechRxiv (https://www.techrxiv.org).

LICENSE

CC BY-NC-SA 4.0

SUBMISSION DATE / POSTED DATE

$22-12-2021 / 28-12-2021$

\section{CITATION}

Nemati, Mahyar; Kim, Yun Hee; Choi, Jinho (2021): Toward Joint Radar, Communication, Computation, Localization, and Sensing in loT. TechRxiv. Preprint. https://doi.org/10.36227/techrxiv.17425469.v1

$\mathrm{DOI}$

10.36227/techrxiv.17425469.v1 


\title{
Toward Joint Radar, Communication, Computation, Localization, and Sensing in IoT
}

\author{
Mahyar Nemati, Member, IEEE, Yun Hee Kim, and Jinho Choi, Senior Member, IEEE
}

\begin{abstract}
Since the 1960s, joint sensing and communication (JSAC) has been proposed as an attractive technique with advantages of enhanced spectral and hardware efficiency along with low latency. However, in those old days, complex transceiver designs hindered the massive adoption of JSAC in many applications. Nevertheless, thanks to advancing wireless technologies in recent years, JSAC has recently attracted substantial attention for a wide range of civil and military applications. In particular, JSAC enables simultaneous sensing and communication functionalities with the full cooperation of both operations in shared resources such as hardware as well as radio resources (i.e., frequency, time, space and so on). Note that sensing functionality is associated with other sub-functionalities of radio-detection-and-ranging (Radar), computation, and localization which are sporadically used terms in the literature. Thus, to generalize the concept and avoid any confusion, we use the term "sensing" in the acronym JSAC as a consistent and general term associated with the other aforementioned sub-functionalities. This paper elaborates on defining sensing and communication operations and then, provides an overview with preliminaries, key latest findings, and state-of-the-art of JSAC. It also explores both existing and emerging Internet of Things (IoT) applications of JSAC. Next, it provides a new classification of JSAC technologies by taking into account not only the existing JSAC technologies but also a diverse range of technologies that allow JSAC to be used for various types of IoT applications. Eventually, this study projects future research directions and challenges of enabling JSAC in IoT.
\end{abstract}

Index Terms-Communication; Computation; Localization; Radar; Sensing; IoT

\section{INTRODUCTION}

With the increasing adoption of fifth-generation (5G) wireless networks, a revolution has been beginning in the smart world where a tremendous number of intelligent devices are connected to each other which is known as the Internet of things (IoT). The IoT is projected to grow significantly over the coming years. The international telecommunication union (ITU) [1] has estimated that more than 19 billion connected devices will be in use worldwide in 2030 resulting in 5016 Exabyte data traffic volume per month; more than $1600 \%$ from 2020 as shown in Figs. 1 (a) and (b). This increase is mostly due to the emergence of massive machine-to-machine (M2M) communication as an intrinsic part of the IoT which is mainly associated with sensing [2]. Hence, two key fundamental functionalities defined for IoT applications are known as (i) communication and (ii) sensing.

Mahyar Nemati and Jinho Choi are with the School of Information Technology, Deakin University, Geelong, VIC 3220, Australia (e-mail: n.mahyar@deakin.edu.au, jinho.choi@deakin.edu.au)

Yun Hee Kim is with department of Electronics and Information Convergence Engineering, Kyung Hee University, Yongin, Republic of Korea (e-mail: yheekim@khu.ac.kr)

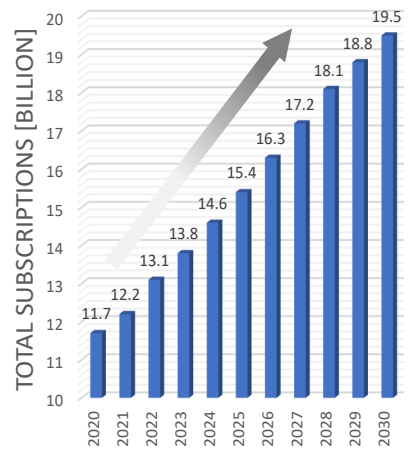

(a)

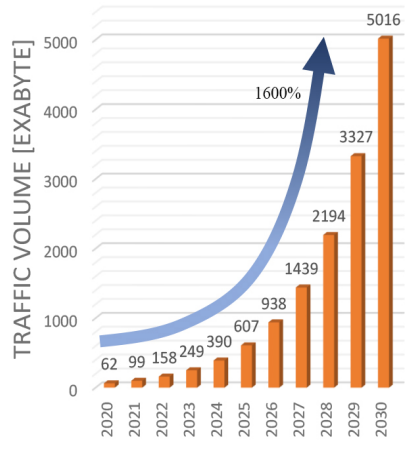

(b)
Fig. 1: Projection of wireless networks by 2030. As the IoT dominance is growing, the number of devices and data traffic volume are increasing exponentially.

\section{A. Preliminaries of Communication \& Sensing}

Communication, in simple terms, allows multiple entities to exchange data/information with each other over wired or wireless channels. Communication happens once there is a specific message generated and transmitted between devices. On the other hand, sensing refers to the detection of various characteristics of objects and environments such as the presence of objects, their shape, location, position gesture, speed of movements, distance, tracking, environmental measurements, channel variations, etc [3]. It is important to note that the main difference between communication and sensing is the data type. In particular, while communication is applied on "desired" messages, sensing is for the detection/estimation of "unknown" features of a network and its entities, as illustrated in Fig. 2. Overall, sensing functionality is associated with other sub-functionalities of radio-detection-and-ranging (radar), computation, and localization which are sporadically used terms in the literature [4]-[6]. Thus, in this paper, we assume that sensing is a consistent and general term that includes the other aforementioned sub-functionalities.

\section{B. Significance of Joint Sensing and Communication}

We expect to see that the majority of data traffic increment in massive $\mathrm{M} 2 \mathrm{M}$ communications is a result of a tremendous number of sensors that will be deployed in emerging applications of smart home/cities, supply chains, retails, wearables, connected health, autonomous cars, etc, where joint/integrated sensing and communication (JSAC) [3] plays a key role in related mobile broadband and massive M2M communications services. In particular, cooperative design of sensing and communication systems with a shared 


\begin{tabular}{|c|c|c|c|}
\hline \multicolumn{4}{|c|}{ LIST OF ABBREVIATIONS } \\
\hline $5 \mathrm{G}$ & Fifth Generation of Wireless Networks & LoRa & Long Range \\
\hline $6 \mathrm{G}$ & Sixth Generation of Wireless Networks & LoS & Line of Sight \\
\hline AMRFC & Advanced Multi-function RF Concept & LTE & Long-Term Evolution \\
\hline AoA & Angle of Arrivals & M2M & Machine-to-Machine communications \\
\hline BackCom & Backscatter Communications & MIMO & Multiple-Input, Multiple-Output \\
\hline CCTV & Closed-Circuit Television & ML & Machine Learning \\
\hline CDMA & Code Division Multiple Access & mmWave & Millimeter-Waves \\
\hline CRSS & Communication and Radar Spectrum Sharing & NASA & National Aeronautics and Space Administration \\
\hline DFRC & Dual-Functional Radar-Communication & NOMA & Non-orthogonal Multiple Access \\
\hline DFT & Discrete-Fourier-Transform & NR & new radio \\
\hline eMBB & Enhanced Mobile Broadband & OFDM & Orthogonal Frequency Division Multiplexing \\
\hline FDD & Frequency-Division-Duplexing & OTFS & Orthogonal Time Frequency Space \\
\hline FMCW & Frequency Modulated Continuous Waveforms & PDSCH & Physical Downlink Shared Channel \\
\hline FOS & $\begin{array}{l}\text { Frequency Modulated Continuous waverorms } \\
\text { Fiber Optic Sensors }\end{array}$ & PUSCH & Physical Uplink Shared Channel \\
\hline GNSS & Global Navigation Satellite Systems & Radar & Radio-detection-and-ranging \\
\hline GSM & $\begin{array}{l}\text { Global Navigation Satellite Systems } \\
\text { Generalized Spatial Modulation }\end{array}$ & RF & Radio Frequency \\
\hline IM & $\begin{array}{l}\text { Generalized Spatial Modulation } \\
\text { Index Modulation }\end{array}$ & RIS & Reconfigurable Intelligent Surfaces \\
\hline IoT & $\begin{array}{l}\text { Index Modulation } \\
\text { Internet of Things }\end{array}$ & RSS & Received-Signal-Strength \\
\hline ITS & $\begin{array}{l}\text { Internet of Things } \\
\text { Intelligent Transportation Systems }\end{array}$ & SINR & Signal-to-Interference-plus-Noise Ratio \\
\hline ITU & Intelligent Transportation Systems & TDD & Time-Division-Duplexing \\
\hline JRC & & TDoA & Time Difference of Arrivals \\
\hline JRCCLS & $\begin{array}{l}\text { Jont Radar Communications } \\
\text { Joint Radar, Communication, Computation, Localization and }\end{array}$ & $\begin{array}{l}\text { THz } \\
\text { UAV }\end{array}$ & $\begin{array}{l}\text { Terahertz } \\
\text { Unmanned Aerial Vehicle }\end{array}$ \\
\hline & Sensing & URLLC & Ultra Reliable Low Latency Communication \\
\hline JSAC & Joint/Integrated Sensing and Communication & $\mathrm{Wi}-\mathrm{Fi}$ & Wireless Fidelity \\
\hline LiDAR & Light Detection and Ranging & WLAN & Wireless Local Area Network \\
\hline
\end{tabular}

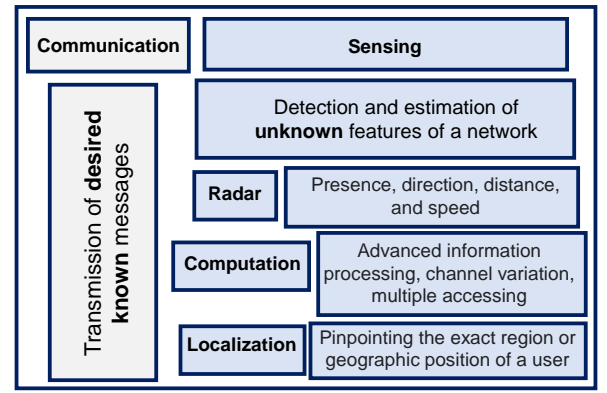

Fig. 2: Communication and sensing functionalities.

spectral and hardware resources is announcing a new era of efficiently utilizing a limited radio-frequency (RF) spectrum [7]. Although the RF sensing brings congestion to the wireless networks, it is advantageous in terms of cost, compact size, less power consumption, spectrum sharing, improved performance, and safety due to enhanced information sharing [7], [8]. In response to these impacts, the literature has made significant progress, with a noticeable increase in interest and activity. Having said that, Fig. 3 shows the total number of publications on communication and sensing systems published between 2010 and Nov. 2021. According to this figure, 78 percent of papers have been published since 2019, indicating the significance of emerging JSAC in recent years.

\section{Background and Motivation}

We now introduce key terminologies in JSAC so as to make the survey paper self-contained. In the view of the literature in both academia and industry, JSAC is introduced with different terminologies such as: joint radar communications (JRC) [4], dual-functional radar-communication (DFRC) [5], and joint radar, communication, computation, localization and

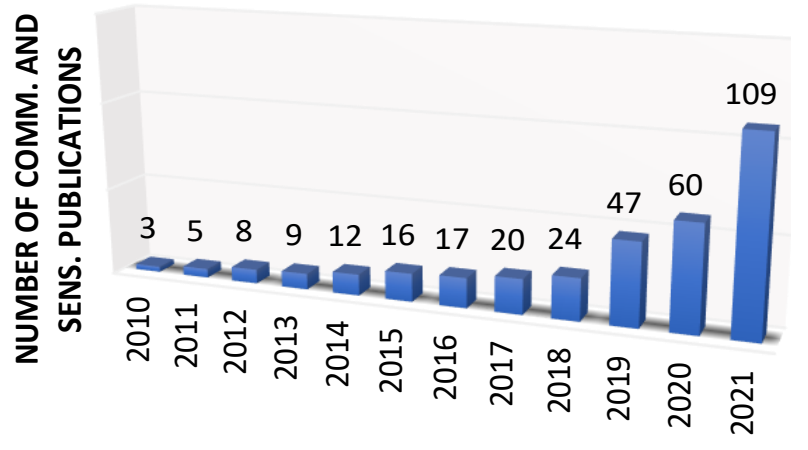

Fig. 3: Communication and sensing publications (accumulative) during 20102021. To collect the papers across different research areas as much as possible, we started by using exact keyword searching on popular scientific databases including Google Scholar, IEEEXplore, ResearchGate and arXiv one by one ${ }^{1}$.

sensing (JRCCLS) [6]. Nevertheless, in this paper, we use the term JSAC as a thorough terminology to refer to all the aforementioned terminologies.

The idea of having multiple functionalities, i.e., sensing and communication, is not new. The primary idea of JSAC technology goes back to the 1960 s when [13] proposed the first JSAC (i.e., DFRC) model to communicate data on the modulated radar pulse intervals simultaneously. Later in the 1970s, national aeronautics and space administration (NASA) proposed a JSAC (i.e., JRC) orbiter Ku-band ${ }^{2}$ system in which the two modes of radar and communication share a wideband multi-function transmitter [14]. It showed how JSAC provides the sensing eyes and the communication ears and

\footnotetext{
${ }^{1}$ The keywords used for searching are "joint communication and sensing", "joint/integrated radar communication", "dual-function communication systems", "passive radar", and "multi-function RF systems".

${ }^{2}$ The Ku-band is the portion of the electromagnetic spectrum in the microwave range of frequencies from $12-18 \mathrm{GHz}$.
} 
TABLE I: Summary of Key Survey Papers in the Areas of JSAC. BackCom: backscatter communications, RIS: reconfigurable intelligent surfaces, ML: machine learning, AI: artificial intelligence.

\begin{tabular}{|c|c|c|c|c|c|c|c|c|c|c|c|c|}
\hline \multirow[t]{2}{*}{ Ref. } & \multirow[t]{2}{*}{ Main Focus } & \multicolumn{6}{|c|}{ Different Sens. \& Comm. paradigms? } & \multicolumn{4}{|c|}{$\begin{array}{l}\text { Compatible Emerging } \\
\text { Technologies for JSAC? }\end{array}$} & \multirow{2}{*}{$\begin{array}{l}\text { JSAC } \\
\text { Classifi- } \\
\text { cation }\end{array}$} \\
\hline & & $\begin{array}{l}\text { Spectrum } \\
\text { sharing }\end{array}$ & BackCom & $\begin{array}{l}\text { Fibre } \\
\text { Optic }\end{array}$ & $\begin{array}{l}\text { Cognitive } \\
\text { radio }\end{array}$ & $\begin{array}{l}\text { Passive } \\
\text { radar }\end{array}$ & JSAC & RIS & mmWave & $\mathrm{THz}$ & $\mathrm{ML} / \mathrm{AI}$ & \\
\hline $\begin{array}{l}\text { This } \\
\text { Sur- } \\
\text { vey }\end{array}$ & $\begin{array}{c}\text { State-of-the-art JSAC } \\
\text { technologies and their } \\
\text { applications }\end{array}$ & $\checkmark$ & $\checkmark$ & $\checkmark$ & $\checkmark$ & $\checkmark$ & $\checkmark$ & $\checkmark$ & $\checkmark$ & $\checkmark$ & $\checkmark$ & $\checkmark$ \\
\hline [3] & $\begin{array}{l}\text { Schemes for JSAC based on } \\
5 \mathrm{G}-6 \mathrm{G} \text { design options }\end{array}$ & $\checkmark$ & $\mathrm{X}$ & $\mathrm{X}$ & $\mathrm{X}$ & $\mathrm{X}$ & $\checkmark$ & $X$ & $\checkmark$ & $X$ & $\checkmark$ & $\mathrm{X}$ \\
\hline [4] & $\begin{array}{l}\text { Perspectives for future } \\
\text { researches of JSAC } \\
\text { technology }\end{array}$ & $\checkmark$ & $\mathrm{X}$ & $\mathrm{X}$ & $\checkmark$ & $\checkmark$ & $\checkmark$ & $X$ & $\mathrm{X}$ & $X$ & $\mathrm{X}$ & $\mathrm{X}$ \\
\hline [5] & $\begin{array}{c}\text { JSAC spectrum sharing } \\
\text { developments }\end{array}$ & $\checkmark$ & $\mathrm{X}$ & $\mathrm{X}$ & $\mathrm{X}$ & $\checkmark$ & $\checkmark$ & $X$ & $\checkmark$ & $\mathrm{X}$ & $\mathrm{X}$ & $X$ \\
\hline [6] & $\begin{array}{l}\text { Review of corresponding key } \\
\text { technical enablers in JSAC }\end{array}$ & $\mathrm{X}$ & $X$ & $\mathrm{X}$ & $\mathrm{X}$ & $\mathrm{X}$ & $\checkmark$ & $\checkmark$ & $\checkmark$ & $X$ & $\checkmark$ & $\mathrm{X}$ \\
\hline [9] & $\begin{array}{l}\text { Perceptive mobile networks } \\
\text { and key technologies }\end{array}$ & $\checkmark$ & $\mathrm{X}$ & $\mathrm{X}$ & $\checkmark$ & $\checkmark$ & $\checkmark$ & $\mathrm{X}$ & $\checkmark$ & $\checkmark$ & $\mathrm{X}$ & $\checkmark$ \\
\hline [10] & $\begin{array}{l}\text { Cellular evolution framework } \\
\text { for JSAC using joint } \\
\text { beamforming }\end{array}$ & $\mathrm{X}$ & $\mathrm{X}$ & $\mathrm{X}$ & $\mathrm{X}$ & $\mathrm{X}$ & $\checkmark$ & $X$ & $\mathrm{X}$ & $\mathrm{X}$ & $\mathrm{X}$ & $\mathrm{X}$ \\
\hline [11] & $\begin{array}{l}\text { Summary of some JSAC } \\
\text { signalling techniques for } \\
\text { spectrum sharing }\end{array}$ & $\checkmark$ & $\mathrm{X}$ & $\mathrm{X}$ & $\checkmark$ & $\checkmark$ & $\checkmark$ & $X$ & $X$ & $\mathrm{X}$ & $\mathrm{X}$ & $\checkmark$ \\
\hline [12] & $\begin{array}{c}\text { Resource management issues } \\
\text { in JSAC and interference } \\
\text { management }\end{array}$ & $\checkmark$ & $\mathrm{X}$ & $\mathrm{X}$ & $\mathrm{X}$ & $\mathrm{X}$ & $\checkmark$ & $\checkmark$ & $\checkmark$ & $\mathrm{X}$ & $\mathrm{X}$ & $X$ \\
\hline
\end{tabular}

voice for a wireless network. NASA continued to develop their Ku-band JSAC system and then, the feasibility of combined functionalities of radar and communication was successfully demonstrated in 1985 [15]. In the 1990s and early 2000s, an advanced multi-function RF concept (AMRFC), i.e., JSAC, was developed by the US Navy as a "proof-of-concept" demonstration with an experimental test-bed. It showed realtime integration of RF functions, such as radar and communications into a single system is technically feasible [16], [17]. In those early stages of JSAC, the efforts were mainly to propose a hardware design, e.g., phased array antennas, for transmissions that are time-shared between communication and radar sensing. Shortly after that and in contrary to those hardware designs for JSAC, spread spectrum with code division multiple access (CDMA) was proposed as a technique to accomplish JSAC profiting from analog chirp filters to produce low correlated up-chirp and low-chirp spread spectrum signals for sensing and communication, respectively [18], [19]. Likewise, another JSAC model based on direct sequence spread spectrum techniques was studied in [20].

Due to the advancement of the technologies in the last decade, researchers have been more interested in JSAC because of its various benefits including enhanced spectrum efficiency and reduced device costs [21]. In [22], an approach for producing JSAC performance bounds was devised based on the information-theoretical analysis. Moreover, there are efforts to exploit the different waveform structures, e.g., orthogonal-frequency-division-multiplexing (OFDM), to piggyback JSAC [18], [23]-[25]. On the other hand, with the massive deployment of multiple-input-multiple-output (MIMO) systems and hybrid analog-digital beamforming, new inventions in JSAC techniques have begun [26]-[28]. In [29], a new JSAC system was introduced where sidelobe control of the transmit beamforming in tandem with waveform diversity enables communication links using the same pulse radar sensing spectrum. In other words, the technique in [29] allows information delivery to multiple communication directions outside the mainlobe of the radar. Furthermore, the work in [30] demonstrated the successful deployment of sparse arrays in JSAC MIMO systems for communication performance enhancement without impacting primary radar sensing functions.

Overall, motivated by the fact that sensing capability is one of the most highlighted new features of future networks [31], JSAC is a very promising area that presents many opportunities and challenges. Further establishment of JSAC within the available infrastructure can deliver a better area coverage as well as a more reliable interconnection between IoT devices while facilitating a multi-static sensory mesh and bringing the sensing capability almost for free [32]. Moreover, the possibility of precise sensing in integration with communication increases with the growing adoption of higher frequency bands in $5 \mathrm{G}$ and $6 \mathrm{G}$, e.g., millimeter-waves (mmWave) and Terahertz (THz) bands.

\section{Scope and Contributions}

This paper aims to provide a high-quality comprehensive survey of JSAC. At a high-level, our primary focus in this work is on providing an overview and the latest findings of the JSAC technology covering its advantages and challenges, as well as new research opportunities for both present and upcoming IoT applications. Moreover, we outline JSAC's different requirements as well as its primary constraint for allowing widespread IoT connectivity. We draw together the aspects of previous works that specifically concern JSAC, 
while simultaneously covering all types of approaches to JSAC that have hitherto been tackled by mobile communication networks. With this as a primary objective, our three main contributions in this paper are outlined as follows:

- This paper defines sensing and communication, overviewing their concepts and properties. It clarifies the differences between various communication and sensing paradigms and provides a comprehensive survey of 115 communication- and sensing-related papers, across various publishing IoT areas such as intelligent transport systems, smart factories/homes/cities, and cellular mobile networks.

- We provide a new classification of JSAC technologies by overviewing not just the existing JSAC connectivity technologies but also diverse state-of-the-art technologies that can be used to provide connectivity for various types of IoT applications.

- We consider the potential of JSAC and examine its distinctive features and benefits over communication-only and sensing-only schemes to address impeding JSAC issues. Based on our understanding, we project future research directions and challenges of enabling JSAC in IoT.

To further elaborate on the contribution of this survey, we summarize the features of existing key survey papers on JSAC in Table I while highlighting the benefits of our survey paper. As given and explained in Table I, we emphasize that despite the existing key surveys, our survey mainly focuses on providing a broad overview on not just the various communication and sensing paradigms but also diverse compatible emerging technologies that can be used to provide JSAC connectivity for various types of IoT applications. In addition, we consider a new classification approach for JSAC technologies with respect to their requirements.

\section{E. Organization}

A high-level view of the structure of the paper is shown in Fig. 4. Section II provides an overview of typical JSAC's use cases, highlighting the benefits of JSAC for IoT applications. Next, Section III presents an overview of different communications and sensing paradigms along with the stateof-the-art of the JSAC and its fundamentals. Then, a new and detailed classification of JSAC technologies along with the recent advancements in JSAC is described in Section IV. After that, Section V elaborates the compatible emerging JSAC technologies. Potential challenges and future research directions are given in Section VI. Finally, Section VII concludes the paper.

\section{Applications And Use CAses of JSAC}

This section elaborates the potentials of JSAC as an exciting combination in a wide range of future IoT network use cases/applications. Indeed, JSAC can enhance user performance where demands for low-cost spectrum-efficient transceivers are increasing. We herein focus on the three main real-life 5G/6G services and use cases: i) Enhanced mobile broadband (eMBB), ii) Intelligent transportation systems

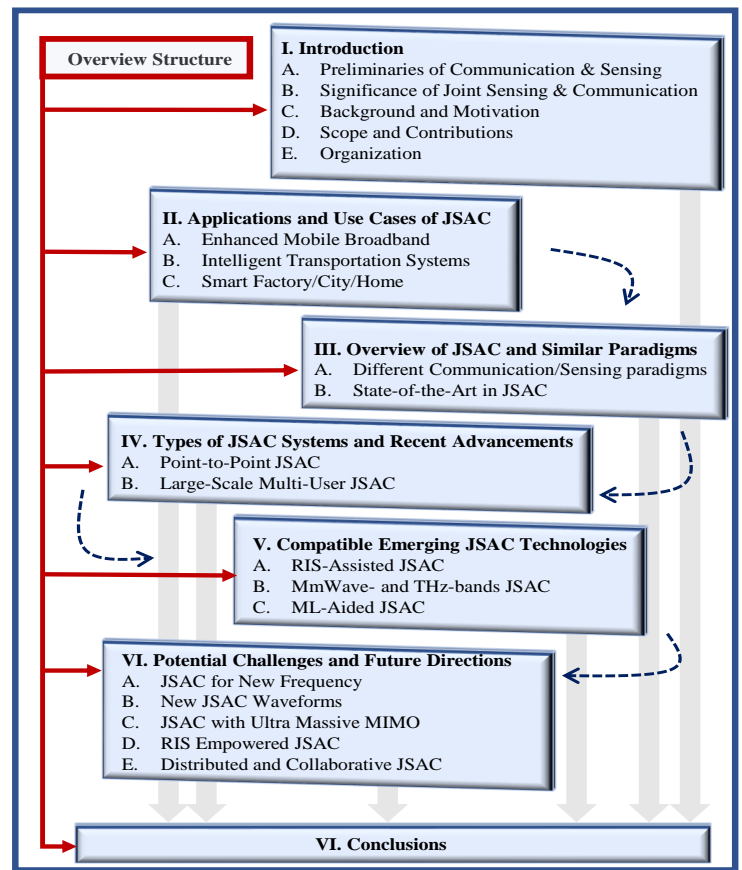

Fig. 4: Organization of the paper and interactions among different sections.

(ITS), and iii) Smart factory/city/home, all of which are now in the deployment phase. As shown in Fig. 5, we briefly review three applications of JSAC along with their requirements in the following.

\section{A. Enhanced Mobile Broadband}

The main objective of JSAC in the existing mobile communication networks is to enhance communication performance through sensing. For instance, obstacles that (temporarily) block the line-of-sight (LoS) link between a mmWave transmitter and receiver can be detected by sensing. Furthermore, sensing functionality can observe the channel gain variation and adjust the communication framework with respect to the desired performance. The rapid network reactions to the sensing input is a crucial factor that highlights the importance of JSAC. In this regard, in [33], deployment of JSAC for an end-to-end communication was investigated and showed that JSAC leads to a huge gain in the transmission rate when the transmitter senses its channel state and conveys a message to the receiver simultaneously. It showed that signal processing techniques are critical for the deployment of JSAC in eMBB systems. Signal processing of such systems can be controlled by exploiting the recent advancements in MIMO, cognitive radio, compressed sensing, and machine learning (ML) tools to reduce the required resources and dynamically allocate them to the eMBB users with low overheads. The work in [7] provides a signal processing perspective of JSAC systems with an emphasis on waveform design. However, there are also challenges for the deployment of JSAC in eMBB. Major challenges are joint waveform design and performance criteria that would optimally trade-off between communication and sensing functionalities. While the accuracy of sensing functionality can be improved in higher frequencies such as 


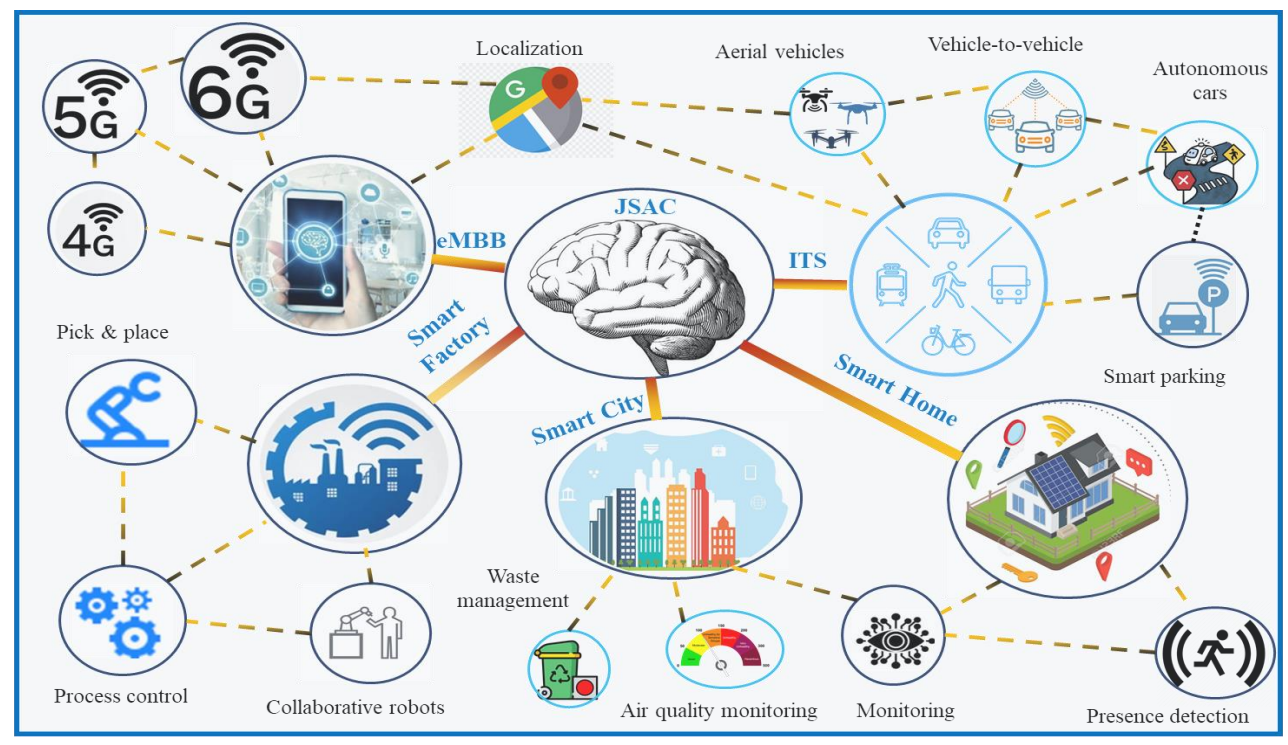

Fig. 5: Illustration of JSAC use cases in IoT applications.

mmWave and $\mathrm{THz}$, its utilization is of interest across the entire frequency range used by cellular networks, beginning at 700 $\mathrm{MHz}$ [32]. For instance, cognitive radio is an application of JSAC whose purpose is to meet the ever-increasing demand for radio spectrum, e.g., in the so-called $700 \mathrm{MHz}$, by dynamically allocating spectrum without interfering with licensed legitimate users [34]. Altogether, sensing can improve cellular communication performance, while various scenarios can be applied to the use cases of eMBB depending on its in-use frequency range.

\section{B. Intelligent Transportation Systems}

It is not exaggerated to say that ITS is the most significant application of JSAC. The use cases of JSAC in ITS includes but not limited to traffic monitoring, autonomous cars, driver identification, localization, velocity measurement and tracking of vehicles, smart parking, etc. In particular, autonomous cars require to constantly sense their surroundings for critical decision makings dealing with human lives. For instance, the accurate speed of a target vehicle can be computed by measuring the Doppler shift and time delays [35]. Furthermore, in the spatial monitoring, reflections of the radio waves are received and processed by the network to generate spatial knowledge of the vehicle's physical environments [32]. These use cases depict the need for large sensor deployments along with the communication transceivers in autonomous vehicles. In a nutshell, both terrestrial and aerial autonomous vehicles including cars and drones are supposed to be equipped with many sensors such as light detection and ranging (LiDAR), cameras, global navigation satellite systems (GNSS), and automotive radar [36]. The utilization of both sensing and communications in autonomous cars stimulates the design of these functionalities in tandem [37]. Interestingly, [38] proposed a JSAC model for autonomous vehicles by embedding information in radar waveforms via index modulation (IM). Besides, [36] used sparse arrays and frequency-modulated continuous waveforms (FMCWs) to enhance the IM-based JSAC systems. Furthermore, in ITS networks, there exists the IEEE 802.11ad standard for mmWave JSAC systems at 60 GHz unlicensed band [25], [39]-[41]. In this mmWave packet transmission system, there are two parts of preamble and message in each packet. Consequently, the message portion is used for communication and the preamble portion is exploited for sensing [42], [43]. Accordingly, various requirements of different JSAC strategies for autonomous vehicles were investigated in [37]. The main requirements are limited antenna size, simplified hardware, low power amplifiers, and interference robustness. Hence, JSAC is supposed to offer potential benefits in performance, size, cost, power consumption, and resilience.

\section{Smart Factory/City/Home}

Communications for industrial IoT (e.g., smart factories) often require the capability of delivering ultra-reliable low latency communication (URLLC) [44], [45] which increases the demands for simultaneous and accurate sensing. Indeed, Industry 4.0 [46] as part of the industrial automation is one of the key applications demanding for a fast and reliable sensor network. Therefore, a practical implementation of a large number of sensing devices in future industrial automation to have continuous communication, computation, and monitoring is needed to meet crucial requirements of energy management, data rate enhancement, and latency minimization. For example, the digital depiction of the real industrial environment necessitates the accurate localization of each moving or stationary object on a factory floor. In particular, precise location estimation surrounding a manufacturing machine can aid in determining where a machine manipulator is placed and if any interfering objects, such as humans, are present inside its intended region of motion [32]. It is possible to do this by utilizing the ubiquitous web of RF signals built primarily to assist communication in an industrial setting. Due to the limited resources, JSAC is an ideal solution for such services. 
To some extent, this set of use cases can be expanded into the emerging smart cities also known as urban sensing/communication technologies [47], in which a digital reproduction of the city can incorporate real-time traffic monitoring, environmental sensing, drone surveillance network, waste management, air quality control and other data to make the city more habitable. The use case relies on localization, computation and monitoring of the city and its entities [6]. Hence, JSAC offers a high potential for integrating radio sensing into large-scale mobile networks in cities, not only to provide quick tracking and localization, but also to go far further and enable the network to see and hear the surroundings. [9].

Eventually, this use case category can involve the immersive smart home [8] use cases to remotely control and manage connected devices in the home such as utilities, smart door locks, doorbells, light bulbs, indoor positioning, gas leak detection, etc. JSAC can provide pervasive and uninterrupted sensing and computing services that creates a platform for smart homes [48]. For example, in this use case family, highresolution imaging is an important use case especially in complete darkness where usual cameras are out of service. Therefore, for instance, in frequencies around $100 \mathrm{GHz}$, the resolution of the sensing image can be obtained below $1 \mathrm{~cm}$ [32]. This can be pretty beneficial in the indoor localization for elderly people and kids tracking [49], [50].

All in all, JSAC systems are divided into two main types: those that do sensing in large-scale networks such as cellular networks and those that perform sensing in point-to-point communication systems [51]. Most of the eMBB use cases can be classified in the first type while the majority of ITS and smart factory/city/home use cases are potentially classified in the second type. We further discuss this classification in section IV.

\section{OVERVIEW OF JSAC AND SIMILAR PARADIGMS}

This section first reviews different communication and sensing paradigms and elaborates their difference with JSAC. Then, it elaborates the stat-of-the-art and different possible sharing protocols and resources in joint communication and sensing

\section{A. Different Communication/Sensing Paradigms}

In general, all communication and sensing paradigms can be classified into two main categories of "communication OR sensing" vs "communication AND sensing", as shown in Fig. 6 , which will be explained in the following.

1) Communication $\boldsymbol{O R}$ Sensing: In this paradigm, which is also known as "Separated Communication and Sensing" [9], communication and sensing are treated as totally separated/different/independent systems divided in all resources, e.g., time, frequency, code, space, etc. Accordingly, communication-only allows two or more entities to exchange messages via any kind of transmission medium based on pre-defined communication protocols; while sensing-only enables data collection, which is the act of perceiving the environment, collecting pertinent information, and delivering

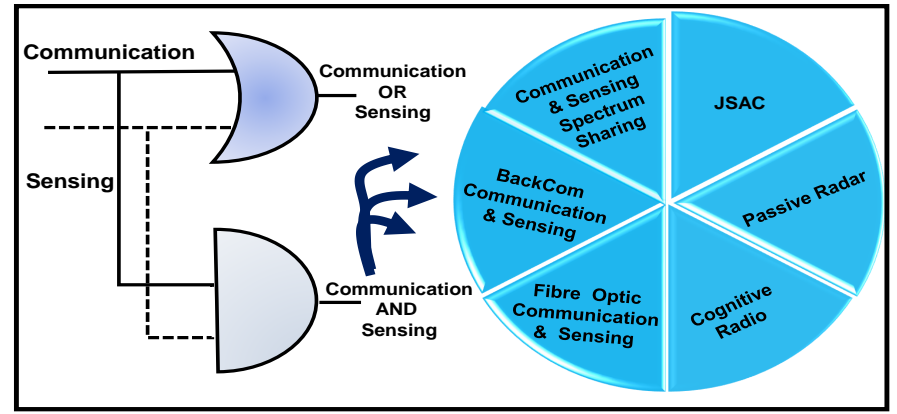

Fig. 6: Classification of different sensing/communication paradigms.

it to the server. Although some communication-only protocols may be used for sensing-only purposes, especially in the phase of data transmission, their functionality and specifications are totally separated and different. For example, in a high-level point of view, an autonomous car contains both sensing-only, e.g., radar sensors, and communication-only modules, e.g., long-term evolution (LTE) transceivers. Radar sensors may use FMCW [52] waveform for sensing purposes while LTE modules use OFDM waveform for data transmission. These two systems not only use different waveforms but are also different in hardware and spectrum. Furthermore, while the interference between these operations is low, the whole system suffers from spectrum inefficiency and complex separated transceiver designs. LiDAR and ultra-sonic sensors are two other examples used for sensing-only purposes with a completely different and separated design from conventional communication-only networks.

2) Communication AND Sensing: In this category, efficiency and coherency are taken into account to combine two functionalities of communication and sensing as much as possible. This leads to enhanced spectrum efficiency and reduced device costs. In the following, we discuss the six main paradigms of "communication AND sensing", as shown in Fig. 6.

- Communication and Sensing Spectrum Sharing: As shown in Fig. 7, this paradigm uses a shared spectrum for both sensing and communication operations. Indeed, it is different from the aforementioned separated paradigm where fixed separated frequency bands are dedicated to communication-only and sensing-only purposes. Here, communication and sensing may use different signals but with the same spectrum utilization, e.g., different waveform designs but at the same spectrum like what was proposed in [53], [54]. In addition, this example is a part of a topic known as communication and radar spectrum sharing (CRSS). According to [5], CRSS was first prompted by the necessity for radar and commercial wireless systems to coexist. CRSS is applied on six main frequency bands of

i) L-band, i.e., 1-2 GHz,

ii) S-band, i.e., 2-4 GHz,

iii) C-band, i.e., 4-8 GHz, 


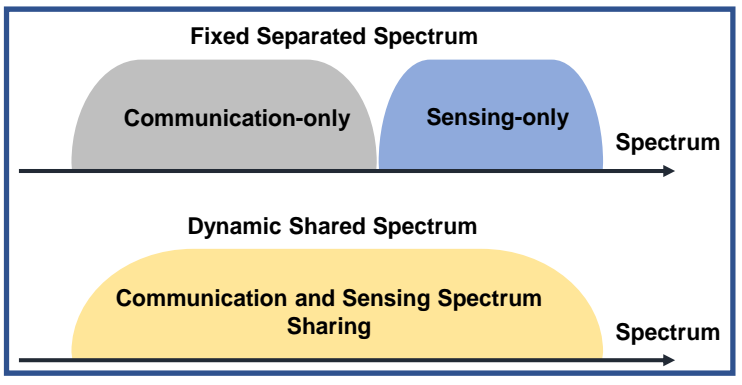

Fig. 7: "Communication and Sensing Spectrum Sharing" vs "separated paradigm"

iv) Ku-band, i.e., $12-18 \mathrm{GHz}$,

v) mmWave-band, i.e., $28-30 \mathrm{GHz}$, and

vi) THz-band, i.e., $0.3 \mathrm{THz}$ to $3 \mathrm{THz}$.

As these bands are projected to be widely assigned to the future $5 \mathrm{G} / 6 \mathrm{G}$ new radio (NR) and Wi-Fi technologies, the interference among sensing and communication in these bands will inevitably increase, causing concerns among military and governmental organizations and spurring study into the issue. For instance, air traffic control radar, frequency-division-duplexing (FDD)LTE and GNSS systems use L-band. The wireless local area networks (WLAN) such as $802.11 \mathrm{~b} / \mathrm{g} / \mathrm{n} / \mathrm{ax} / \mathrm{y}$ and $3.5 \mathrm{GHz}$ time-division-duplexing (TDD)-LTE systems are deployed in S-band. C-band is mainly occupied by $802.11 \mathrm{a} / \mathrm{h} / \mathrm{j} / \mathrm{n} / \mathrm{p} / \mathrm{ac} / \mathrm{ax}$ and Ku-band is widely used for satellite communications and NASA's tracking data relay satellite. The use cases of mmWave-band include but not limited to $802.11 \mathrm{ad} /$ ay WLAN protocols, 5G NR, vehicle to vehicle communications. Finally, the utilization of THz-band is an emerging topic for wireless indoor communication systems and fiber-equivalent wireless links, to name a few.

In general, although the spectrum efficiency in this model is higher than the separated paradigm, it suffers from interference. Moreover, complex signal processing is required along with precise cooperation between terminals [9]. Furthermore, the study in [9] expanded this paradigm into a more general framework called "Coexisting Communication and Sensing" which does not limit the system to the spectrum sharing but extends the sharing idea toward other mutual resources such as antennas, time, spreading codes in CDMA, etc.

- Backscatter Communication and Sensing: Backscatter communication (BackCom) technology usually contains a passive/semi-passive tag, e.g., RFID systems [55], which receives a RF signal (either dedicated or ambient RF signal). Next, the tag modulates the RF signal and sends its own data over it. Since the activation of these passive tags depends on the sensing of the interrogation signal, BackCom is considered as a JSAC technology with a shared hardware of the BackCom tags/transceivers for both sensing and communication operations as shown in Fig. 8. Ambient BackCom [56] which uses ambient signals takes one step further for spectrum efficiency as it uses the same spectrum as ambient RF signals for

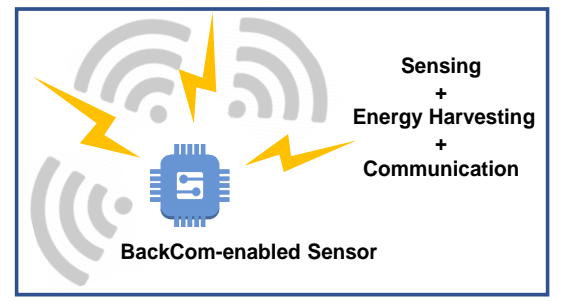

Fig. 8: BackCom-enabled sensors are empowered by sensing, energy harvesting and communication.

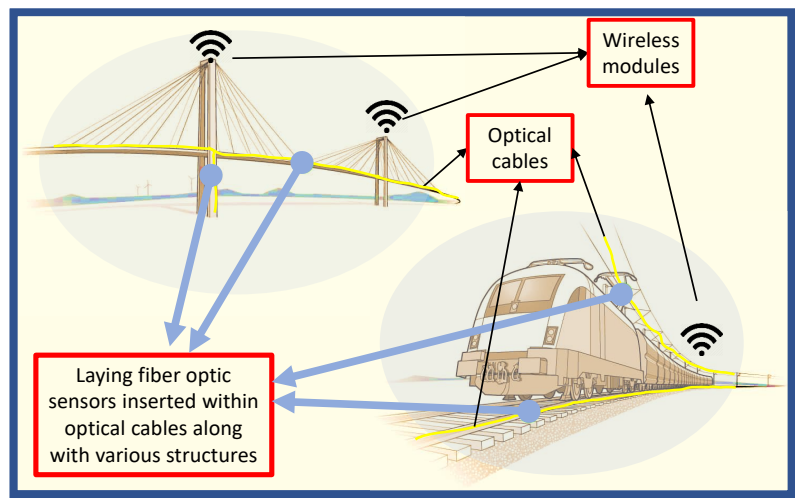

Fig. 9: Fiber optic communication and sensing in cooperation with wireless modules.

short-range communication purposes. For instance, in a large-scale overview, [57] proposed a JSAC model for the willingness to sense a large number of the RFID tags, e.g., attached to the attendees in a stadium, without guaranteed successful communication for each tag. This showed that the sensing in BackCom JSAC can mitigate the excessive signal processing imposed by a large number of tags communicating with a reader. The idea in [58] proposed a cooperative ambient BackCom for JSAC that the reader recovers information not only from the RF source, i.e., communication, but also from the sensing tag, i.e., sensing. In general, JSAC has a great potential for enhancing the BackCom system performance where BackCom tags require to constantly evaluate their surroundings RF signals for detecting the interrogation signals and sensing the high power radio waves for efficient energy harvesting to support a continuous uninterrupted communication.

- Fiber Optic Communication and Sensing: Fiber optic sensors (FOSs) are a type of JSAC system containing optical sensors connected to a network of optical cables. The sensors are prefabricated along with the optical cables while cables can be used for regular data transmission. Hence, the FOSs are one of the most common wired JSAC systems that are passive, low weight, and have low maintenance cost and are widely used for structural health and geotechnical monitoring and instrumentation as shown in Fig. 9. Although many wireless technologies are to measure things, FOSs are more advantageous wired JSAC systems in terms of the high reliability and accuracy in demanding environments. In terms of functionality, FOSs are appropriate for parameter measurements such as strain, deformations, forces, temperature, 


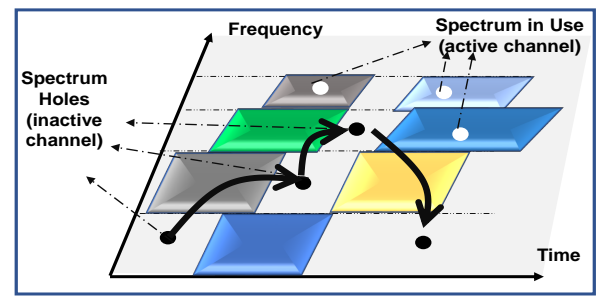

Fig. 10: Dynamic spectrum accessing in cognitive radio.

accelerations, rotations, electric fields, and so on, which are super beneficial not only in smart city applications but also in smart factories and homes. The FOSs are classified into four main categories: i) point-based sensors, e.g., Fabry-Perot [59], [60], ii) Quasi distributed sensors (multiplexed), e.g., fiber-Bragg-grating (FBG) [61], ii) Longbase sensors [62], iv) Distributed sensors, e.g., Brillouin and Roman scattering [63]. For instance, the long-base FOSs can be used to monitor column lifetime variations in a building. The distributed model can measure the strain and temperature in every meter along the fiber cable and is very useful to detect cracks and leaks and localize them rather than only measuring them. FOS technology is a high-resolution sensing paradigm and have the ability to replace conventional sensors for civil applications and particularly can be used for those cases where using vibrating sensors is difficult for non-destructive testing. It is also usable for long distances since fiber cables are cheaper than copper cables and high power complex wireless transmitters. Moreover, no electrical power is needed in remote areas and there is no electromagnetic interference. However, the communication can be significantly affected by the sensing priority as the communication is limited into a special optical fiber direction where sensors are needed to be deployed. Besides, wireless links are often mobile and quicker than optical networks in terms of pure communication speed. Hence, as shown in Fig. 9, the coexistence of FOSs and wireless modules are beneficial.

- Cognitive Radio: Cognitive radio is another type of "Communication AND Sensing" that allows a transceiver to sense active and inactive communication channels and distinguish them. Then, the transceiver utilizes the available inactive channels while avoiding those that are already in use for its communication purposes [64], as shown in Fig. 10. This paradigm is super beneficial for eMBB uses cases especially in the future $5 \mathrm{G} / 6 \mathrm{G}$ NR technologies, where there are billions of cellular users but they are not always active. Therefore, the inactive channels can be sensed and used dynamically by a large number of users, i.e., dynamic spectrum sharing. Cognitive radio is an advantageous technology to enhance spectrum efficiency and minimize interference. However, in busy networks, there might be a huge delay imposed on the network as the transceivers need to wait until they sens desired inactive channels. Besides, there are some technical issues on Cognitive Radio-based IoT Systems

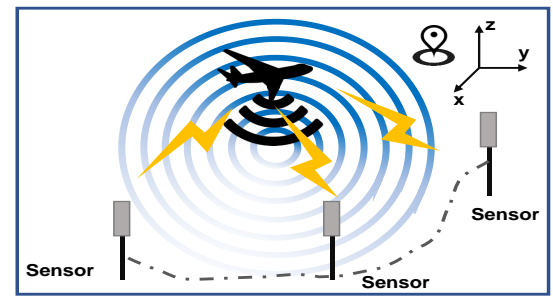

Fig. 11: Passive radar localization using multiple passive sensors.

which are elaborated in [65]. For example, the hardware designs of cognitive radio transceivers always require multi-band antennas which increases the cost for low-cost $\&$ low-power IoT devices. Moreover, there is a security problem [66] since cognitive radio technology provides more opportunities for attackers than standard wireless networks.

- Passive Radar: Passive radar is a popular sensing technology that includes a special passive sensing receiver with the ability to listen to special radio signals and extract the desired sensing information. Passive sensing is widely used for passive localization methods, as shown in Fig. 11, like received-signal-strength (RSS), time-difference-of-arrivals (TDoA) and angle-of-arrival (AoA) methods [67]. Other use cases of passive sensing include but not limited to air traffic control, weather observation, remote sensing of the environment, aircraft and ship navigation. This paradigm does not require any modification in the existing networks and profits from a high spectrum efficiency compared to the previous aforementioned paradigms. However, it required a dedicated sensing receiver, reception time is not clear and there is a large latency. As a result sensing capability is restricted and multi-user detection is challenging [9]. To some extent, passive sensing, which uses dispersed signals from non-cooperative communication systems, might be considered a subset of CRSS technology [5].

- JSAC In this paradigm, researchers not only research spectrum-related issues but also look at whether sensing and communication can share the utmost number of resources to help relieve resources scarcity and reduce device costs. The desired paradigm of JSAC aims at using similar signals for both sensing and communication with the highest possible spectral efficiency, low latency and ultra-high reliability. It investigates the fully shared transmitter and largely shared receiver designs [68]. As a result, precise optimization is required to be done on mutual resources such as waveform, time, frequency, etc.

Overall, there are different sensing and communication paradigms in both optical, e.g., LiDAR and cameras, and RF bands, e.g., ultra-wideband, cellular, and Wi-Fi, with different advantages and weaknesses. For instance, although opticalbased technologies have a high resolution and reliability, radiobased techniques are often more resistant to environmental influences such as bad illumination. Next, we further discuss the state-of-the-art of JSAC. 


\section{B. State-of-the-Art in JSAC}

The state-of-the-art of JSAC is to simultaneously sense the surrounding environment and deliver data packets. Compared to the conventional existing sensing technologies like videobased monitoring or even more advanced technologies like LiDAR systems which need LoS and clear links, the emerging JSAC is supposed to be mounted on RF communication signals and monitor the surroundings even under NLoS environments. The capability of RF signals to propagate in NLoS, dark, and different weather conditions provides an opportunity to have reliable sensing utilizing the existing mobile communications infrastructures [32]. Furthermore, with the massive increase in the number of mobile phone users and volume of data traffic by 2030, shown in Figs. 1 (a) and (b), the spectrum scarcity becomes a challenging problem. Even, a significant portion of the current in-use spectrum is dedicated to mobile communication users. Nevertheless, as time goes on, more automated sensing systems are emerging, like autonomous cars. Hence, the capability of JSAC systems in efficient spectrum utilization for both sensing and communication purposes is beneficial. Even, by moving toward higher frequency bands in $5 \mathrm{G}$ and $6 \mathrm{G}$ systems, high-resolution sensing becomes more feasible. In many places, there might be a limitation for installing some conventional monitoring systems like surveillance cameras for a variety of reasons, e.g., privacy concerns. Here, JSAC systems using RF transmissions impinge on privacy less than cameras. On the other hand, considering the huge number of cellular base-stations and mobile users in urban areas these days, JSAC can perform better in terms of sensing because of the densification of reference nodes [69].

Designing a general waveform to piggyback both sensing and communication signals is an important topic. For instance, conventional utilization of spread spectrum with CDMA appends two sensing and communication signals causing volatile amplitude signal and get distorted if a nonlinear amplifier is used [18]. Therefore, OFDM with its flexible and specific structure was taken into account with a constant-envelope OFDM symbol for a specific JSAC approach in [70] to avoid non-constant amplitude signals. In [71], a variety of possible approaches based on classical phase-coded waveforms and MIMO systems were investigated. Furthermore, a new fullduplex JSAC system was proposed in [72] with an optimized OFDM waveform for low latency data transmission. It utilized the empty subcarriers within the OFDM frame for sensing to improve the spectrum efficiency of the system while enabling dual functionality. The authors also addressed how the peakto-average power ratio of the waveform can be controlled and minimized along the optimization process. Moreover, IMbased techniques [73], [74] have recently become popular models for both OFDM waveforms and MIMO systems where indices of subcarriers or antennas can be allocated for sensing purposes.

\section{TyPes of JSAC Systems AND RECENT ADVANCEMENTS}

This section further elaborates the detailed technologies and recent advancements of the JSAC along with a novel

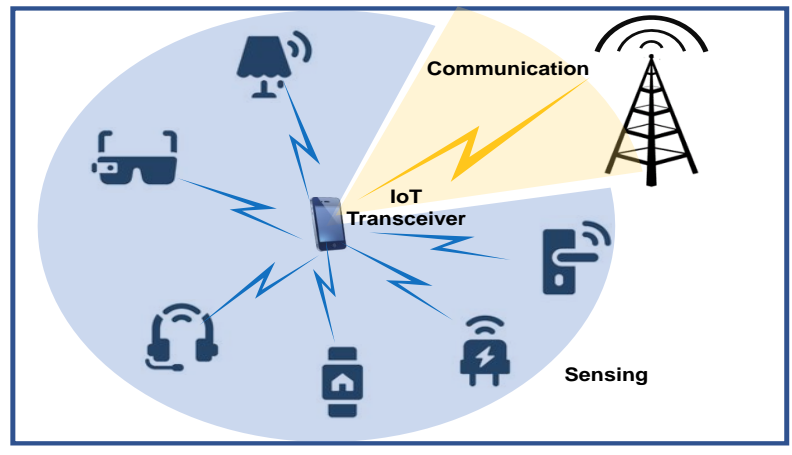

Fig. 12: Point-to-point JSAC is a single multi-functional prototype enabled for both sensing and communication purposes of single IoT transceiver.

classification of JSAC systems available in the literature. It contains two main classes of i) Point-to-Point JSAC, ii) LargeScale Multi-User JSAC which are discussed in the following.

\section{A. Point-to-Point JSAC}

Point-to-point JSAC aims at designing a transceiver platform, as shown in Fig. 12, to piggyback both sensing and communication functionalities for special purposes. In [75], a hardware prototype for a spatial IM-based JSAC system was proposed with respect to two coexisting sensing and communications subsystems. It employs generalized spatial IM and distributed transceiver antenna components across sensing and communication subsystems based on the sent message, resulting in enhanced communication rates by integrating more data bits in the antenna selection indices. By assessing the statistical properties of the transmit beam patterns and coexisting waveforms, it showed that co-designing these subsystems can result in significant improvements in performance, power consumption, cost, and size. In other words, profiting from spatial IM, we can have better communication performance and the spatial agility induced by the spatial IM transmission improves the angular resolution in the sensing and reduces the sidelobe level in the transmit beam pattern compared to techniques utilizing fixed antenna allocations. In [9], a perceptive point-to-point mobile network framework was proposed with radio sensing capability used to get information from received mobile signals from items of interest in the area around the radio transceivers. It concluded that three types of signals in 5G NR have the properties that can be used for sensing such as i) reference signals, ii) synchronization signal blocks, and iii) data payloads. Additionally, different forms of signal embedding techniques for JSAC systems have been described in [76], where JSAC was considered a solution to the spectrum congestion problem. Furthermore, [77]-[79] discussed how the physical downlink shared channel (PDSCH) and physical uplink shared channel (PUSCH) can be used for sensing purposes. In [80], a LoRa-assisted point-to-point JSAC model was proposed for a remote structural health monitoring system of bolted joints in smart city environments. This work leverages long-range (LoRa) technology for both communication and sensing operations to enable wide communication coverage and long life structural health monitoring. 


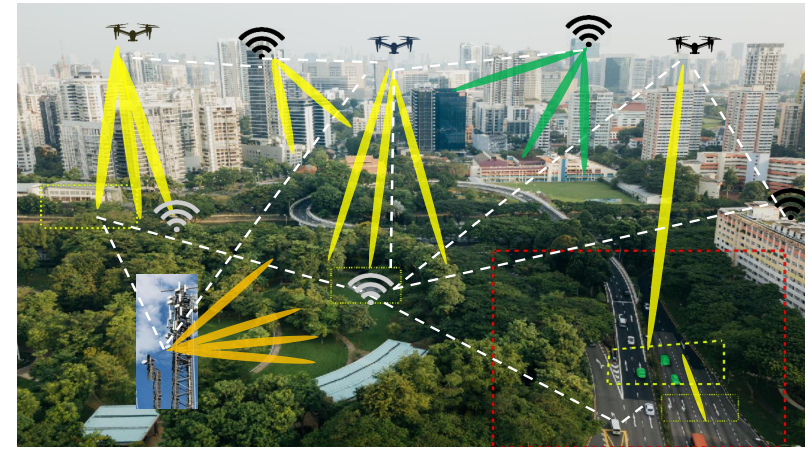

Fig. 13: Existing cellular infrastructure can piggyback the large-scale multiuser JSAC technologies.

\section{B. Large-Scale Multi-User JSAC}

Suppose that there are multiple communication-only users along with sensing-only targets like a cellular base-station communicating with mobile users while localizing aerial vehicles. Here, the cellular base-station should be empowered by a large-scale multi-user JSAC architecture for joint communication and localization purposes, as shown in Fig. 13. In [81], a large-scale framework was developed for a novel perceptive mobile/cellular network that integrates radar sensing function into the mobile communication network. This JSAC concept employed a single system platform that allows both downlink and uplink sensing as well as the exchange of sent signals with communications. However, sensing becomes more challenging within the rich-multipath mobile networks and requires more sophisticated signal processing techniques. Here, compressive sensing can be considered as a useful tool for sensing performance along with the OFDMA for multiple accessing in multi-user scenarios. In [82], a simple multi-access scenario for a JSAC system was purposed with dynamic estimationand data-rate for sensing or communication, respectively; depending on the importance of each operation. Additionally, in [83], a joint transmit beamforming model was proposed for a multi-user JSAC where the system transmits the weighted sum of independent radar waveforms and communication symbols, forming multiple beams toward the radar targets and the communication receivers. It necessitates additional modification of the MIMO radar transmit beamforming weighting coefficients while ensuring that the signal-to-interference-plus-noise ratio (SINR) at each communication user is greater than a certain threshold. However, inter-user and sensing interference is crucial to be re-assessed in this JSAC model because of the radar performance degradation compared to the original radaronly systems. Furthermore, within the context of large-scale monitoring areas for applications such as smart farming, the authors in [84] evaluated a coordinated sensing coverage of a large-scale unmanned aerial vehicles (UAV) communication network based on the average perceptual interference of UAVs and the communication capacity limits. Another large-scale sensory network was proposed in [85] which uses grant-free non-orthogonal multiple-accessing (NOMA) as an emerging JSAC technique in massive M2M communications for reducing signaling overhead.

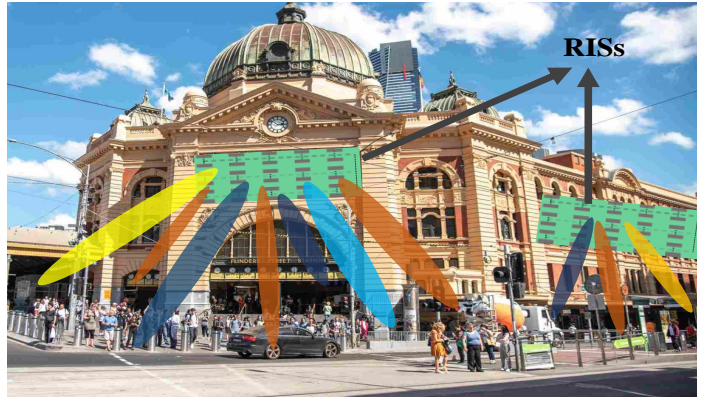

Fig. 14: RIS can be hung on walls and enable sensing while enhancing communication performance at the same time.

\section{Compatible Emerging JSAC Technologies}

This section mainly focuses on the emerging technologies that have a great potential to be compatible with JSAC to achieve situational awareness. It covers emerging technologies such as reconfigurable intelligent surfaces (RIS), mmWave and $\mathrm{THz}$ communications, and ML-aided JSAC as three of the most popular emerging technologies in future IoT connectivity schemes.

\section{A. RIS-Assisted JSAC}

RIS is an emerging technology that can manipulate impinging electromagnetic waves to benefit wireless users [86], [87]. A brief illustration of RIS is shown in Fig. 14 as a surface with a large number of passive sensors that are capable of changing impinging signal characteristics such as phase, amplitude, and polarization. It is evident that accurate sensing of the impinging wave attributes plays a vital role in programming intelligent surfaces to obtain a required performance [88]. Hence, JSAC is crucial not only for spectral- \& hardware efficiency but also for RIS performance improvement. Furthermore, while such RISs have been designed primarily for communication reasons, they can bring significant gains in terms of performance, energy consumption, and cost for localization and mapping [89].

Recently, [31] investigated the wireless sensing potential of RIS walls and demonstrated that, in addition to the attention given to RIS in terms of communication characteristics, it can provide a high-resolution radio image, i.e., digital twin, of the propagation environment. This is due to the fact that RIS can be positioned close to the desired sensing space and the high-resolution is provided by densely spaced small sensors distributed over a wide surface. Consequently, [31] utilized image processing and ML tools to enhance the sensing performance of an RIS. In addition, RIS can act as a secondary transmitter sending its own sensed information, such as environmental data from embedded sensors, to the end-user over the same ambient impinging RF signal from a transmitter while assisting the primary transmission from the desired transmitter to its receiver. This later model using a cooperative single antenna ambient BackCom tag was proposed in [58]. Utilization of compressive sensing tools to construct the channels at all the RIS elements while RIS is in the communication phase was investigated in [90]. This occurred as a result of the commitment of a portion of RIS 
components for compressed sensing and deep learning purposes to forecast the ideal RIS reflection coefficients directly from sampled channel knowledge. This helps a simultaneous channel sensing and also shows the status of the environment along with the position of the transmitter and receiver. Besides, [88] advocated using simple one-antenna power measurements to conduct compressed sensing of the impinging wave and communicate with chosen transceivers. In other words, the RIS may be configured to perform both wave detection and wave modification simultaneously. The model in [91] studied a novel RIS setup for simultaneously localizing a target and serving a user. Then, the new arrangement was utilized to improve the JSAC's capabilities in mmWave frequencies. It divided the RIS-elements into distinct divisions for sensing and communication functions in an adaptive manner. The sensing elements are responsible for localizing the target, while the remaining elements maintain a robust communication link with the user. In addition, [89] examined case studies of RIS-based localization and mapping services where JSAC is extremely profitable. Furthermore, [92] discussed how RIS improves joint communication and localization performance and demonstrated how the RIS phase profile can be adjusted for JSAC. Another work in [93] used a semi-passive RIS by inserting a small set of active sensors distributed in the surface to support a JSAC model for simultaneous channel sensing and communication. It proposed a multi-scale enhanced deep residual channel estimator to improve generalization and fitting capabilities by increasing the entire network's flexibility. It is capable of striking a balance between system complexity and estimating performance. Furthermore, [94] studied a RISassisted JSAC model for minimizing channel estimation errors. The research results in [95] showed that RISs can also play an important role in radar applications, revealing the first basic trade-offs and major difficulties. It looked into a target detection challenge in a sensing architecture where RIS can offer an extra echo of the target to the radar. The work in [96] focused on Industry 4.0 applications and addressed the potential of RIS-aided JSAC for not only high throughput and efficient multiplexing of wireless links, but also to provide a high-resolution representation of the propagation environment. To be specific, in a smart factory environment, the RIS can be positioned close to the phenomenon being sensed, while the high-resolution is provided by densely spaced small sensors dispersed across a vast region. In a nutshell, since RIS can be regarded as a radio picture of the environment, a RISassisted JSAC approach can identify whether an industrial robot deviates from a specified route using computer vision and ML tools [96].

Overall, the emergence of RIS provides a range of capabilities including sensing, computation and localization along with its communication functionality. It assists the concept of JSAC in wireless channels and can be used in a wide variety of applications such as smart homes, factories, cities, etc.

\section{B. MmWave- and THz-bands JSAC}

Both mmWave and $\mathrm{THz}$ frequencies have the potential to give significant wireless communication capacity performance

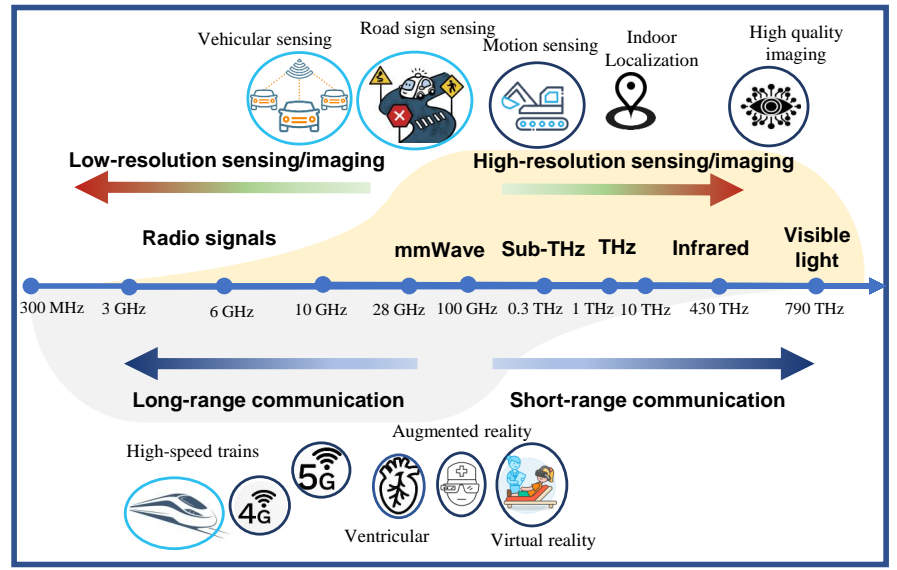

Fig. 15: MmWave and $\mathrm{THz}$ spectrum with respect to communication and sensing functionalities.

benefits and allow high-resolution environment sensing if correctly applied thanks to their vast amount of accessible bandwidth [97]. However, there are some challenging issues such as highly uncertain and dynamic wireless channels in these high frequencies. To be specific, due to the inherent short communication range and high sensitivity to blocking and molecule absorption, these high-frequency channel constraints result in unreliable intermittent communications. As a result, such barriers may jeopardize the mmWave and $\mathrm{THz}$ band's promise of high-speed communications and highresolution sensing capabilities. The study in [97] described and thoroughly explored seven distinct defining aspects of $\mathrm{THz}$ wireless systems showing how to re-engineer wireless systems as we know them now in order to accommodate $\mathrm{THz}$ bands and their distinct contexts. It depicted that $\mathrm{THz}$ systems benefit from quasi-opticality and may convert every communication issue into a sensing opportunity, therefore contributing to a new generation of adaptable JSAC wireless systems capable of performing various functionalities beyond simple communications. In [98], the authors took a stride in this course by developing a $\mathrm{THz}$ sensing prototype with millimeter range imaging decision whilst taking into account the aperture size limitation of standard cellphones. Indeed it developed $\mathrm{THz}$ JSAC for $6 \mathrm{G}$ and beyond as a convergence of sensing, imaging, and positioning. Moreover, [21] elaborated the compatibility of OFDM systems with THz JSAC by inventing a non-uniform multi-wideband OFDM waveform. Furthermore, [99] evaluated the autonomous vehicles as a potential real-life utilization domain of joint high data-rate communications and high-resolution radar sensing operating in the mmWave- and THz-bands. For instance, it showed how steerable mmWave antenna arrays are used for both sensing and communication functionalities. The work in [100] proposed a unified activity detection and channel estimation for both mmWave and $\mathrm{THz}$ wideband massive access. In [101], a new model took the advantage of RISs into account for joint channel sensing and high data-rate communication in $\mathrm{THz}$ MIMO communication systems. Another work in [102] designed a joint discrete-Fourier-transform (DFT) spread OFDM waveform and deep learning reception architecture 
for THz JSAC systems. On the other hand, [103] developed a different waveform design for TDD-based JSAC systems in $\mathrm{THz}$ communications in which it can achieve ultra-highresolution sensing only with cost-efficient analog-to-digital converters with low sampling rate. It showed the proposed waveform is a robust technique against strong Doppler shift effects of received target detection signals. In the utilization of THz bands in environmental monitoring applications, [104] investigated a climate change sensing methodology through the absorption of $\mathrm{THz}$ signals by certain gases as a new strategy of air quality monitoring with $\mathrm{THz}$ JSAC systems. In [105], a JSAC downlink $\mathrm{THz}$ approach using NOMA was proposed where a joint beamforming-power-bandwidth allocation and communication was employed. It improved the sum-rate performance of the NOMA system compared to traditional orthogonal multiple access systems. Furthermore, in [5], a mmWave JSAC system architecture based on massive MIMO and hybrid analog-digital beamforming structure was presented. It made use of the TDD frame structure to integrate sensing and communication in the following scenarios: target detection, channel estimation, communication, and tracking. It also demonstrated that the proposed JSAC system can be implemented on a single mmWave base-station.

In general, beyond the aforementioned benefits of mmWave and $\mathrm{THz}$ bands, these systems can take the advantage of RISs, low frequency bands, and ML tools to ensure robust system performance as well [97]. Both mmWave and $\mathrm{THz}$ bands are to support a wide variety of services as shown in Fig. 15.

\section{ML-Aided JSAC}

An unrepentant degree of freedom can be provided using ML recent developments in JSAC systems to open up new possibilities and improvements in both sensing and communication operations. ML can efficiently examine dynamic patterns in the wireless environment at a far lower complexity than the traditional optimization approaches [2]. Within the context of large-scale JSAC systems, UAV networks and their adoption in various communication- and sensing-based applications require dynamic and intelligent system configurations. Hence, the ML framework can provide the ability of learning for UAVs automation. The work in [106] addressed the different issues when UAVs are utilized and identified ML approaches utilized to improve the different design and functional elements such as channel modeling, resource management, location, and security. In [107], densification of intelligent devices is taken into account and different ML algorithms in sensor data analytics within the agricultural ecosystem were reviewed. The work in [108] discussed the development of ML techniques and proposed an intelligent system with the ability to perceive the environment, evaluate the resources/spectrum and learn how to adapt system configurations to make the most use of the given resources. The study in [109] reviewed signal processing and ML Techniques for THz JSAC systems. Overall, ML is a strong tool that can optimize efficient wireless resource utilization in JSAC since resource allocation optimization issues are typically too difficult to model owing to dynamic wireless settings.

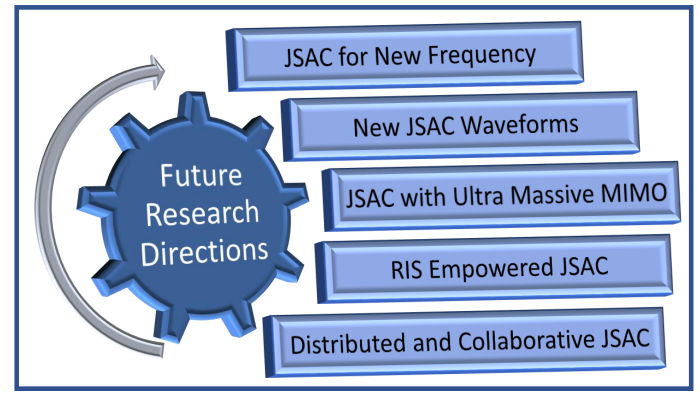

Fig. 16: Future Research directions

\section{Future ReseARCH DiRECTIONS}

Future IoT networks based on $6 \mathrm{G}$ and beyond are expected to operate not only at sub-6 GHz supporting long-range connectivity but also at a higher carrier frequency such as mmWave, sub-THz, and $\mathrm{THz}$ bands, coveting an unoccupied ultra-wide band. In this context, we expect that various JSAC concepts and methodologies would appear by introducing and combining the emerging technologies surveyed in Section V. The important and emerging topics to be studied in the future are identified as follows.

\section{A. JSAC for New Frequency:}

JSAC in sub-THz and $\mathrm{THz}$ bands can be designed from the scratch less resorting to the existing standards. Thus, various multi-purpose JSAC platforms can be developed with their unique features at various trade-offs in sensing and communication performance. We believe that the informationtheoretical analysis on the JSAC under new channel characteristics reveals the intrinsic trade-off in sensing and communication that leads to novel system design criteria. New waveforms appropriate for given channel models as well as new applications should be designed under the system design criterion, accompanied by signal processing at transmitter and receivers. In addition, infrastructures such as ultra-massive antennas for pencil-like beamforming and RISs for shaping the channel propagation environments favorable to JSAC should be studied to cope with severe path losses in sub- $\mathrm{THz}$ and $\mathrm{THz}$ bands. In the overall system design, ML and deep learning would be key enablers in sensing and data detection as well as controlling the beam pattern and RIS reflection/absorption patterns.

\section{$B$. New JSAC Waveforms:}

Design of a new waveform for the new frequency bands is crucial to cope with the peculiar channel characteristics of a higher frequency and hardware impairments such as non-linear effect of a high power amplifier. Given that a new waveform beyond OFDM signaling is promising at a new frequency, it will lead to tremendous reformation of transmitting and receiving platforms accompanied by new algorithms. A genuine JSAC can be accomplished by designing a waveform common to sensing and communication with a predictable trade-off between sensing accuracy and 
communication performance. It is rather easier for the pointto-point JSAC but is rather intricate for the large-scale JSAC generating multiple signals for massive IoT devices. In the latter case, a superposition of sensing and communications like NOMA would be provide a controllable performance trade-off between sensing and communication. One of such waveforms can be found in the recent work in [103], where a superposition of multiple Zadoff-Chu (ZC) sequences in multiple subbands was proposed for the $\mathrm{THz}$ band JSAC. New waveforms can be designed not only in the time-frequency domain but also in the delay-Doppler domain for robustness to the Doppler shifts for new frequency and vehicular networks. The delayDoppler domain design includes orthogonal-time-frequencyspace (OTFS) [110] but not limited to. We believe that various JSAC waveforms should be explored according to sensing purposes and communication requirements that are dependent on IoT application and deployment scenarios.

\section{JSAC with Ultra Massive MIMO:}

Ultra massive MIMO that is more feasible at a higher frequency is expected to extend the sensing and communication coverage as well as serve more number of IoT devices with a larger degree of freedom in the spatial domain. However, the utmost complexity of ultra massive MIMO and its required signal processing hindered its practical implementation. Recently, metasurface antennas with either continuous or discrete meta elements are attracting considerable attention to realize ultra massive MIMO at low hardware complexity and cost [111], [112]. It is also expected that the directly controlled metasurface antennas could provide holographic beamforming and modulation and sense the holographic image of nearby IoT devices at a higher new frequency. In this context, it will be challenging but interesting research direction to feed and control metasurface antennas to construct multiple beams of different missions such as sensing and communication and to precept their environment. We also believe that ML and deep learning play key role in the efficient control of the metasurface and analysis of the holographic image perceived by the antennas.

\section{RIS Empowered JSAC:}

Most research studies on RISs have focused on RIS-assisted communication, where RISs take a role of a relay changing radio environments favorable to communication. A simple scenario of JSAC with RISs simply adds sensing functions to the base-station whilst remaining the RISs to reflect the signals as instructed by the base-station. If active sensing is incorporated at the base-station, the sensed parameters at the base-station such as AoA and multipath delays can improve the channel estimation and its related operation. In this context, one of interesting subjects to be investigated in the near future is how sensing information can be incorporated to optimize transmit beamforming and RIS reflections coefficients.

On the other hand, not only reflecting the incident signals, an RIS is made to absorb the signals for passive sensing by installing sensors sparsely in the RIS or by adopting a metasurface capable of both reflection and absorbance. Since passive RIS sensing can be exploited for various use cases such as the channel parameter estimation or localization and tracking of IoT devices, it is required to develop a JSAC protocol for each use case and provide the sensing algorithms using low-resolution RIS sensing data. Additionally, allocation of RIS elements to sensing and communication, optimization of the RIS reflection coefficients, and deployment optimization of multiple RISs including UAV-RIS are required to be investigated for simultaneous sensing and communication offering a diverse trade-off. We would like to note again that ML and deep learning are a powerful tool for sensing algorithms and optimization problems in JSAC with RISs.

\section{E. Distributed and Collaborative JSAC:}

As observed earlier, not only the base-stations but also multiple RISs and UAVs could be deployed in the future IoT networks to serve ubiquitous IoT devices that are capable of sensing, computation, and communication [113]. These constituting JSAC entities not only generate heterogeneous sensory data but also correlated data. It is also an important research subject how to analyze and communicate the sensory data distributed in the network to obtain a targeted feature and desirable information in various applications, e.g., regression [?]. For data without any privacy issue, the sensory data can be collected at a base-station or a relay for the centralized analysis and learning through efficient communication protocols. For sensory data with a privacy issue, the JSAC entities could train their local models for federated learning [114]. In this context, one of a research subject for the IoT networks with JSAC would be joint design of communication protocols and learning models to share the sensory data or learning model while meeting the network requirements such as communication latency and sensing accuracy.

\section{CONCLUSIONS}

This survey paper reviewed JSAC, which is a promising technology for $6 \mathrm{G}$, to go beyond communication and to offer multi-functional capabilities for a wide range of IoT use cases such as eMBB services, smart factories/homes/cities, and ITS. It was shown that the integration of sensing and communication has a significant potential to enhance the system's performance while also supporting new and intriguing sensing and/or communication capabilities to various applications. Moreover, different available combinations of communication and sensing functionalities were categorized and described based on the shared resources among sensing and communication operations. JSAC was discussed as a new model to look at how the utmost number of resources can be shared among sensing and communication to relieve resources scarcity and reduce device costs. In addition, a new classification for JSAC systems was provided based on the following two main classes: i) point-to-point JSAC and ii) large-scale multi-user JSAC. The former mainly focused on transceiver and waveform design, while the later concerned more about large-scale deployment of JSAC devices, signal processing and multiple access techniques. Furthermore, compatible emerging technologies like RIS, mmWave \& THz bands, and AI/ML 
aided JSAC were discussed. Eventually, we shed light on the distinguishing characteristics and possible benefits of JSAC over communication-only and sensing-only systems, as well as providing insights into its prospective research directions.

\section{ACKNOWLEDGEMENT}

This work was supported by Australian Research Council (ARC) Discovery 2020 Funding, under grant number DP200100391.

\section{REFERENCES}

[1] M. Z. Chowdhury, M. Shahjalal, S. Ahmed, and Y. M. Jang, "6g wireless communication systems: Applications, requirements, technologies, challenges, and research directions," IEEE Open Journal of the Communications Society, vol. 1, pp. 957-975, 2020.

[2] J. Ding, M. Nemati, C. Ranaweera, and J. Choi, "IoT Connectivity Technologies and Applications: A Survey," IEEE Access, vol. 8, pp. 67 646-67 673, 2020.

[3] T. Wild, V. Braun, and H. Viswanathan, "Joint design of communication and sensing for beyond $5 \mathrm{~g}$ and $6 \mathrm{~g}$ systems," IEEE Access, vol. 9, pp. 30 845-30857, 2021.

[4] Z. Feng, Z. Fang, Z. Wei, X. Chen, Z. Quan, and D. Ji, "Joint radar and communication: A survey," China Communications, vol. 17, no. 1, pp. 1-27, 2020.

[5] F. Liu, C. Masouros, A. P. Petropulu, H. Griffiths, and L. Hanzo, "Joint Radar and Communication Design: Applications, State-of-the-Art, and the Road Ahead," IEEE Transactions on Communications, vol. 68, no. 6 , pp. 3834-3862, 2020.

[6] H. Wymeersch, D. Shrestha, C. M. De Lima, V. Yajnanarayana, B. Richerzhagen, M. F. Keskin, K. Schindhelm, A. Ramirez, A. Wolfgang, M. F. De Guzman et al., "Integration of communication and sensing in 6g: a joint industrial and academic perspective," in 2021 IEEE 32nd Annual International Symposium on Personal, Indoor and Mobile Radio Communications (PIMRC). IEEE, 2021, pp. 1-7.

[7] K. V. Mishra, M. Bhavani Shankar, V. Koivunen, B. Ottersten, and S. A. Vorobyov, "Toward Millimeter-Wave Joint Radar Communications: A Signal Processing Perspective," IEEE Signal Processing Magazine, vol. 36, no. 5, pp. 100-114, 2019.

[8] Q. Huang, H. Chen, and Q. Zhang, "Joint Design of Sensing and Communication Systems for Smart Homes," IEEE Network, vol. 34, no. 6, pp. 191-197, 2020.

[9] J. A. Zhang, M. L. Rahman, K. Wu, X. Huang, Y. J. Guo, S. Chen, and J. Yuan, "Enabling joint communication and radar sensing in mobile networks-A survey," IEEE Communications Surveys \& Tutorials, 2021.

[10] Q. Qi, X. Chen, C. Zhong, and Z. Zhang, "Integrated sensing, computation and communication in b5g cellular internet of things," IEEE Transactions on Wireless Communications, vol. 20, no. 1, pp. 332-344, 2020.

[11] L. Zheng, M. Lops, Y. C. Eldar, and X. Wang, "Radar and communication coexistence: An overview: A review of recent methods," IEEE Signal Processing Magazine, vol. 36, no. 5, pp. 85-99, 2019.

[12] N. C. Luong, X. Lu, D. T. Hoang, D. Niyato, and D. I. Kim, "Radio Resource Management in Joint Radar and Communication: A Comprehensive Survey," IEEE Communications Surveys \& Tutorials, 2021.

[13] R. M. Mealey, "A method for calculating error probabilities in a radar communication system," IEEE Transactions on Space Electronics and Telemetry, vol. 9, no. 2, pp. 37-42, 1963.

[14] R. Cager, D. LaFlame, and L. Parode, "Orbiter Ku-band integrated radar and communications subsystem," IEEE Transactions on Communications, vol. 26, no. 11, pp. 1604-1619, 1978.

[15] J. Griffin, J. Kelley, A. Steiner, H. Vang, W. Zrubek, and G. Huth, "Shuttle ku-band communications/radar technical concepts," in Space Shuttle Tech. Conf, Pt. 2 p 757-766(SEE N 85-16937 08-12), 1985.

[16] P. K. Hughes and J. Y. Choe, "Overview of advanced multifunction RF system (AMRFS)," in Proceedings 2000 IEEE International Conference on Phased Array Systems and Technology (Cat. No. 00TH8510). IEEE, 2000, pp. 21-24.

[17] G. C. Tavik, C. L. Hilterbrick, J. B. Evins, J. J. Alter, J. G. Crnkovich, J. W. de Graaf, W. Habicht, G. P. Hrin, S. A. Lessin, D. C. Wu et al., "The advanced multifunction RF concept," IEEE transactions on microwave theory and techniques, vol. 53, no. 3, pp. 1009-1020, 2005 .
[18] C. W. Rossler, E. Ertin, and R. L. Moses, "A software defined radar system for joint communication and sensing," in 2011 IEEE RadarCon (RADAR), 2011, pp. 1050-1055.

[19] G. N. Saddik, R. S. Singh, and E. R. Brown, "Ultra-wideband multifunctional communications/radar system," IEEE Transactions on Microwave Theory and Techniques, vol. 55, no. 7, pp. 1431-1437, 2007.

[20] X. Shaojian, C. Bing, and Z. Ping, "Radar-communication integration based on DSSS techniques," in 2006 8th international Conference on Signal Processing, vol. 4. IEEE, 2006.

[21] Y. Wu, F. Lemic, C. Han, and Z. Chen, "A non-uniform multi-wideband ofdm system for terahertz joint communication and sensing," in 2021 IEEE 93rd Vehicular Technology Conference (VTC2021-Spring), 2021, pp. 1-5.

[22] D. W. Bliss, "Cooperative radar and communications signaling: The estimation and information theory odd couple," in 2014 IEEE Radar Conference. IEEE, 2014, pp. 0050-0055.

[23] A. Hassanien, B. Himed, and B. D. Rigling, "A dual-function mimo radar-communications system using frequency-hopping waveforms," in 2017 IEEE Radar Conference (RadarConf). IEEE, 2017, pp. 17211725 .

[24] A. Ahmed, Y. D. Zhang, and Y. Gu, "Dual-function radarcommunications using QAM-based sidelobe modulation," Digital Signal Processing, vol. 82, pp. 166-174, 2018.

[25] P. Kumari, S. A. Vorobyov, and R. W. Heath, "Adaptive virtual waveform design for millimeter-wave joint communication-radar," IEEE Transactions on Signal Processing, vol. 68, pp. 715-730, 2019.

[26] E. Fishler, A. Haimovich, R. Blum, D. Chizhik, L. Cimini, and R. Valenzuela, "MIMO radar: An idea whose time has come," in Proceedings of the 2004 IEEE Radar Conference (IEEE Cat. No. 04CH37509). IEEE, 2004, pp. 71-78.

[27] X. Zhang, A. F. Molisch, and S.-Y. Kung, "Variable-phase-shift-based RF-baseband codesign for MIMO antenna selection," IEEE Transactions on Signal Processing, vol. 53, no. 11, pp. 4091-4103, 2005.

[28] F. Liu, C. Masouros, A. Li, T. Ratnarajah, and J. Zhou, "MIMO radar and cellular coexistence: A power-efficient approach enabled by interference exploitation," IEEE Transactions on Signal Processing, vol. 66, no. 14, pp. 3681-3695, 2018.

[29] A. Hassanien, M. G. Amin, Y. D. Zhang, and F. Ahmad, "Dual-function radar-communications: Information embedding using sidelobe control and waveform diversity," IEEE Transactions on Signal Processing, vol. 64, no. 8, pp. 2168-2181, 2015.

[30] X. Wang, A. Hassanien, and M. G. Amin, "Dual-function MIMO radar communications system design via sparse array optimization," IEEE Transactions on Aerospace and Electronic Systems, vol. 55, no. 3, pp. 1213-1226, 2018.

[31] C. J. Vaca-Rubio, P. Ramirez-Espinosa, K. Kansanen, Z.-H. Tan, E. De Carvalho, and P. Popovski, "Assessing Wireless Sensing Potential With Large Intelligent Surfaces," IEEE Open Journal of the Communications Society, vol. 2, pp. 934-947, 2021.

[32] H. Andersson Y, "Unpacking joint communication and sensing in 6G," www.ericsson.com, 10 2021. [Online]. Available: https://www.ericsson.com/en/blog/2021/10/joint-sensingand-communication- $6 \mathrm{~g}$

[33] M. Ahmadipour, M. Wigger, and M. Kobayashi, "Joint Sensing and Communication over Memoryless Broadcast Channels," in 2020 IEEE Information Theory Workshop (ITW), 2021, pp. 1-5.

[34] M. R. Bambal and A. Satmohankar, "Review on cognitive cellular network," 2016.

[35] T. Jia, K. Ho, H. Wang, and X. Shen, "Localization of a moving object with sensors in motion by time delays and Doppler shifts," IEEE Transactions on Signal Processing, vol. 68, pp. 5824-5841, 2020.

[36] D. Ma, N. Shlezinger, T. Huang, Y. Liu, and Y. C. Eldar, "FRaC: FMCW-Based Joint Radar-Communications System via Index Modulation," IEEE Journal of Selected Topics in Signal Processing, 2021.

[37] _ - "Joint radar-communication strategies for autonomous vehicles: Combining two key automotive technologies," IEEE signal processing magazine, vol. 37, no. 4, pp. 85-97, 2020.

[38] D. Ma, N. Shlezinger, T. Huang, Y. Shavit, M. Namer, Y. Liu, and Y. C. Eldar, "Spatial modulation for joint radar-communications systems: Design, analysis, and hardware prototype," IEEE Transactions on Vehicular Technology, vol. 70, no. 3, pp. 2283-2298, 2021.

[39] G. Duggal, S. Vishwakarma, K. V. Mishra, and S. S. Ram, "Dopplerresilient 802.11 ad-based ultrashort range automotive joint radarcommunications system," IEEE Transactions on Aerospace and Electronic Systems, vol. 56, no. 5, pp. 4035-4048, 2020. 
[40] A. Tang, S. Li, and X. Wang, "Self-Interference-Resistant IEEE 802.11 ad-Based Joint Communication and Automotive Radar Design," IEEE Journal of Selected Topics in Signal Processing, 2021.

[41] A. Ali, N. Gonzalez-Prelcic, R. W. Heath, and A. Ghosh, "Leveraging sensing at the infrastructure for mmWave communication," IEEE Communications Magazine, vol. 58, no. 7, pp. 84-89, 2020.

[42] E. Grossi, M. Lops, L. Venturino, and A. Zappone, "Opportunistic radar in IEEE 802.11 ad networks," IEEE Transactions on Signal Processing, vol. 66, no. 9, pp. 2441-2454, 2018.

[43] P. Kumari, J. Choi, N. González-Prelcic, and R. W. Heath, "IEEE 802.11 ad-based radar: An approach to joint vehicular communicationradar system," IEEE Transactions on Vehicular Technology, vol. 67 , no. 4, pp. 3012-3027, 2017.

[44] A. Aijaz and M. Sooriyabandara, "The Tactile Internet for Industries: A Review," Proceedings of the IEEE, vol. 107, no. 2, pp. 414-435, 2019.

[45] J. Ding, M. Nemati, S. R. Pokhrel, O.-S. Park, J. Choi, and F. Adachi, "Enabling grant-free URLLC: An overview of principle and enhancements by massive MIMO," IEEE Internet of Things Journal, 2021.

[46] Z. Shi, Y. Xie, W. Xue, Y. Chen, L. Fu, and X. Xu, "Smart factory in Industry 4.0," Systems Research and Behavioral Science, vol. 37, no. 4, pp. 607-617, 2020.

[47] B. Harris et al., "Data-driven Internet of Things systems and urban sensing technologies in integrated smart city planning and management," Geopolitics, History, and International Relations, vol. 13, no. 1 , pp. 53-63, 2021.

[48] W. Liu, K. Huang, X. Zhou, and S. Durrani, "Next generation backscatter communication: systems, techniques, and applications," EURASIP Journal on Wireless Communications and Networking, vol. 2019, no. 1, pp. 1-11, 2019.

[49] N. Thakur and C. Y. Han, "Pervasive Activity Logging for Indoor Localization in Smart Homes," in 2021 4th International Conference on Data Science and Information Technology, 2021, pp. 246-255.

[50] S. K. Pandey, S. Sonia, T. Semwal, and S. B. Nair, "Smart Patch: An IoT based Anti Child-Trafficking Solution," in 2020 IEEE International Conference on Internet of Things and Intelligence System (IoTaIS). IEEE, 2021, pp. 194-200.

[51] J. A. Zhang, F. Liu, C. Masouros, R. W. Heath Jr, Z. Feng, L. Zheng, and A. Petropulu, "An Overview of Signal Processing Techniques for Joint Communication and Radar Sensing," arXiv preprint arXiv:2102.12780, 2021

[52] T. Instruments, "AWR1843 Single-Chip 77-and 79-GHz FMCW Radar Sensor," 2017.

[53] Paracha, Kashif Nisar and Abdul Rahim, Sharul Kamal and Soh, Ping Jack and Khalily, Mohsen, "Wearable Antennas: A Review of Materials, Structures, and Innovative Features for Autonomous Communication and Sensing," IEEE Access, vol. 7, pp. 56694-56712, 2019.

[54] A. Ayyar and K. V. Mishra, "Robust communications-centric coexistence for turbo-coded OFDM with non-traditional radar interference models," in 2019 IEEE Radar Conference (RadarConf). IEEE, 2019, pp. $1-6$.

[55] M. Nemati, M. Soltani, J. Ding, and J. Choi, "Subcarrier-Wise Backscatter Communications Over Ambient OFDM for Low Power IoT," IEEE Transactions on Vehicular Technology, vol. 69, no. 11, pp. $13229-13242,2020$

[56] M. Nemati, J. Ding, and J. Choi, "Short-range ambient backscatter communication using reconfigurable intelligent surfaces," in 2020 IEEE Wireless Communications and Networking Conference (WCNC). IEEE, 2020, pp. 1-6.

[57] M. Nemati, H. Takshi, and V. Shah-Mansouri, "Tag estimation in RFID systems with capture effect," in 2015 23rd Iranian Conference on Electrical Engineering. IEEE, 2015, pp. 368-373.

[58] G. Yang, Q. Zhang, and Y.-C. Liang, "Cooperative Ambient Backscatter Communications for Green Internet-of-Things," IEEE Internet of Things Journal, vol. 5, no. 2, pp. 1116-1130, 2018.

[59] X. Zhou, Q. Yu, and W. Peng, "Fiber-optic Fabry-Perot pressure sensor for down-hole application," Optics and Lasers in Engineering, vol. 121, pp. 289-299, 2019.

[60] N. Hussain, M.-J. Jeong, J. Park, and N. Kim, "A broadband circularly polarized fabry-perot resonant antenna using a single-layered PRS for 5G MIMO applications," IEEE Access, vol. 7, pp. 42 897-42 907, 2019.

[61] S. Chettouh, A. El-Akrmi, H. Triki, and Y. Hamaizi, "Spectral properties of nonlinearly chirped fiber Bragg gratings for optical communications," Optik, vol. 147, pp. 163-169, 2017.

[62] F. Q. Kareem, S. R. Zeebaree, H. I. Dino, M. A. Sadeeq, Z. N. Rashid, D. A. Hasan, and K. H. Sharif, "A survey of optical fiber communications: challenges and processing time influences," Asian Journal of Research in Computer Science, pp. 48-58, 2021.

[63] L. Hu, L. Sheng, J. Yan, L. Li, M. Yuan, F. Sun, F. Nian, L. Li, J. Liu, S. Zhou et al., "Simultaneous Measurement of Distributed Temperature and Strain through Brillouin Frequency Shift Using a Common Communication Optical Fiber," International Journal of Optics, vol. 2021, 2021.

[64] W. S. H. M. W. Ahmad, N. A. M. Radzi, F. Samidi, A. Ismail, F. Abdullah, M. Z. Jamaludin, and M. Zakaria, "5G technology: Towards dynamic spectrum sharing using cognitive radio networks," IEEE Access, vol. 8, pp. 14460-14 488, 2020.

[65] F. A. Awin, Y. M. Alginahi, E. Abdel-Raheem, and K. Tepe, "Technical issues on cognitive radio-based Internet of Things systems: A survey," IEEE access, vol. 7, pp. 97 887-97908, 2019.

[66] A. Haldorai and A. Ramu, "Security and channel noise management in cognitive radio networks," Computers \& Electrical Engineering, vol. 87, p. 106784, 2020.

[67] M. Nemati, T. Baykas, and J. Choi, "Performance of TDOA and AOA Localization Techniques for Different Base-Stations Topologies," in 2019 13th International Conference on Signal Processing and Communication Systems (ICSPCS), 2019, pp. 1-7.

[68] M. L. Rahman, J. A. Zhang, K. Wu, X. Huang, Y. J. Guo, S. Chen, and J. Yuan, "Enabling joint communication and radio sensing in mobile networks-a survey," arXiv preprint arXiv:2006.07559, 2020

[69] H. Chae, "Integrated Communication and Sensing for B5G/6G-Part 1: Key Drivers and Applications," offino, 2021. [Online]. Available: https://1djhuc37mndh310ow71uejzf-wpengine.netdna-ssl.com/wpcontent/uploads/2021/11/OFNO_14768-White-Sheet-M2_111821b.pdf

[70] D. Garmatyuk, J. Schuerger, Y. Morton, K. Binns, M. Durbin, and J. Kimani, "Feasibility study of a multi-carrier dual-use imaging radar and communication system," in 2007 European Microwave Conference. IEEE, 2007, pp. 1473-1476.

[71] C. Sturm and W. Wiesbeck, "Waveform Design and Signal Processing Aspects for Fusion of Wireless Communications and Radar Sensing," Proceedings of the IEEE, vol. 99, no. 7, pp. 1236-1259, 2011

[72] S. D. Liyanaarachchi, T. Riihonen, C. B. Barneto, and M. Valkama, "Optimized waveforms for $5 \mathrm{~g}-6 \mathrm{~g}$ communication with sensing: Theory, simulations and experiments," IEEE Transactions on Wireless Communications, pp. 1-1, 2021.

[73] E. Basar, M. Wen, R. Mesleh, M. Di Renzo, Y. Xiao, and H. Haas, "Index modulation techniques for next-generation wireless networks," IEEE access, vol. 5, pp. 16693-16746, 2017.

[74] A. M. Jaradat, J. M. Hamamreh, and H. Arslan, "OFDM with hybrid number and index modulation," IEEE Access, vol. 8, pp. 55 042-55 053 , 2020.

[75] D. Ma, N. Shlezinger, T. Huang, Y. Shavit, M. Namer, Y. Liu, and Y. C. Eldar, "Spatial modulation for joint radar-communications systems: Design, analysis, and hardware prototype," IEEE Transactions on Vehicular Technology, vol. 70, no. 3, pp. 2283-2298, 2021.

[76] A. Hassanien, M. G. Amin, E. Aboutanios, and B. Himed, "Dualfunction radar communication systems: A solution to the spectrum congestion problem," IEEE Signal Processing Magazine, vol. 36, no. 5 , pp. 115-126, 2019.

[77] Y. Gao, Z. Deng, Y. Zhang, S. Sun, and Z. Li, "Mobile phone passive positioning through the detection of uplink signals for search and rescue," Sensors, vol. 19, no. 20, p. 4526, 2019.

[78] K. Shamaei, J. Khalife, and Z. M. Kassas, "Performance characterization of positioning in LTE systems," in Proceedings of the 29th international technical meeting of the satellite division of the institute of navigation (ION GNSS+ 2016), 2016, pp. 2262-2270.

[79] L. Chen, X. Zhou, F. Chen, L.-L. Yang, and R. Chen, "Carrier Phase Ranging for Indoor Positioning with 5G NR Signals," IEEE Internet of Things Journal, 2021

[80] M. Sidorov, P. V. Nhut, Y. Matsumoto, and R. Ohmura, "Lora-based precision wireless structural health monitoring system for bolted joints in a smart city environment," IEEE Access, vol. 7, pp. 179 235-179 251, 2019.

[81] M. L. Rahman, J. A. Zhang, X. Huang, Y. J. Guo, and R. W. Heath, "Framework for a perceptive mobile network using joint communication and radar sensing," IEEE Transactions on Aerospace and Electronic Systems, vol. 56, no. 3, pp. 1926-1941, 2020.

[82] A. R. Chiriyath, B. Paul, and D. W. Bliss, "Radar-communications convergence: Coexistence, cooperation, and co-design," IEEE Transactions on Cognitive Communications and Networking, vol. 3, no. 1, pp. 1-12, 2017.

[83] X. Liu, T. Huang, N. Shlezinger, Y. Liu, J. Zhou, and Y. C. Eldar, "Joint transmit beamforming for multiuser MIMO communications and 
MIMO radar," IEEE Transactions on Signal Processing, vol. 68, pp. 3929-3944, 2020.

[84] X. Chen, Z. Feng, Z. Wei, F. Gao, and X. Yuan, "Performance of Joint Sensing-Communication Cooperative Sensing UAV Network," IEEE Transactions on Vehicular Technology, vol. 69, no. 12, pp. 15545 $15556,2020$.

[85] Y. Du, B. Dong, W. Zhu, P. Gao, Z. Chen, X. Wang, and J. Fang, "Joint Channel Estimation and Multiuser Detection for Uplink GrantFree NOMA," IEEE Wireless Communications Letters, vol. 7, no. 4, pp. 682-685, 2018.

[86] M. Nemati, J. Park, and J. Choi, "RIS-Assisted Coverage Enhancement in Millimeter-Wave Cellular Networks," IEEE Access, vol. 8, pp. 188 171-188 185, 2020.

[87] M. Nemati, B. Maham, S. R. Pokhrel, and J. Choi, "Modeling RIS Empowered Outdoor-to-Indoor Communication in mmWave Cellular Networks," IEEE Transactions on Communications, pp. 1-1, 2021.

[88] C. Liaskos, A. Tsioliaridou, A. Pitilakis, G. Pirialakos, O. Tsilipakos, A. Tasolamprou, N. Kantartzis, S. Ioannidis, M. Kafesaki, A. Pitsillides, and I. Akyildiz, "Joint Compressed Sensing and Manipulation of Wireless Emissions with Intelligent Surfaces," in 2019 15th International Conference on Distributed Computing in Sensor Systems (DCOSS), 2019, pp. 318-325.

[89] H. Wymeersch, J. He, B. Denis, A. Clemente, and M. Juntti, "Radio Localization and Mapping With Reconfigurable Intelligent Surfaces: Challenges, Opportunities, and Research Directions," IEEE Vehicular Technology Magazine, vol. 15, no. 4, pp. 52-61, 2020.

[90] A. Taha, M. Alrabeiah, and A. Alkhateeb, "Enabling Large Intelligent Surfaces With Compressive Sensing and Deep Learning," IEEE Access, vol. 9, pp. 44304-44 321, 2021.

[91] R. Sankar, B. Deepak, and S. P. Chepuri, "Joint Communication and Radar Sensing with Reconfigurable Intelligent Surfaces," arXiv preprint arXiv:2105.01966, 2021.

[92] A. Elzanaty, A. Guerra, F. Guidi, and M.-S. Alouini, "Reconfigurable Intelligent Surfaces for Localization: Position and Orientation Error Bounds," IEEE Transactions on Signal Processing, vol. 69, pp. 53865402, 2021.

[93] Y. Jin, J. Zhang, X. Zhang, H. Xiao, B. Ai, and D. W. K. Ng, "Channel Estimation for Semi-Passive Reconfigurable Intelligent Surfaces With Enhanced Deep Residual Networks," IEEE Transactions on Vehicular Technology, vol. 70, no. 10, pp. 11083-11088, 2021.

[94] J. Lin, G. Wang, R. Fan, T. A. Tsiftsis, and C. Tellambura, "Channel estimation for wireless communication systems assisted by large intelligent surfaces," arXiv preprint arXiv:1911.02158, 2019.

[95] S. Buzzi, E. Grossi, M. Lops, and L. Venturino, "Radar Target Detection Aided by Reconfigurable Intelligent Surfaces," IEEE Signal Processing Letters, vol. 28, pp. 1315-1319, 2021.

[96] C. J. Vaca-Rubio, P. Ramirez-Espinosa, R. J. Williams, K. Kansanen, Z.-H. Tan, E. de Carvalho, and P. Popovski, "A primer on large intelligent surface (lis) for wireless sensing in an industrial setting," arXiv preprint arXiv:2006.06563, 2020.

[97] C. Chaccour, M. N. Soorki, W. Saad, M. Bennis, P. Popovski, and M. Debbah, "Seven defining features of terahertz $(\mathrm{THz})$ wireless systems: A fellowship of communication and sensing," arXiv preprint arXiv:2102.07668, 2021

[98] O. Li, J. He, K. Zeng, Z. Yu, X. Du, Y. Liang, G. Wang, Y. Chen, P. Zhu, W. Tong, D. Lister, and L. Ibbotson, "Integrated Sensing and Communication in 6G A Prototype of High Resolution THz Sensing on Portable Device," in 2021 Joint European Conference on Networks and Communications 6G Summit (EuCNC/6G Summit), 2021, pp. 544-549.

[99] V. Petrov, G. Fodor, J. Kokkoniemi, D. Moltchanov, J. Lehtomaki, S. Andreev, Y. Koucheryavy, M. Juntti, and M. Valkama, "On Unified Vehicular Communications and Radar Sensing in Millimeter-Wave and Low Terahertz Bands," IEEE Wireless Communications, vol. 26, no. 3 , pp. 146-153, 2019.

[100] X. Shao, X. Chen, C. Zhong, and Z. Zhang, "Joint activity detection and channel estimation for $\mathrm{mmW} / \mathrm{THz}$ wideband massive access," in ICC 2020-2020 IEEE International Conference on Communications (ICC). IEEE, 2020, pp. 1-6.

[101] X. Ma, Z. Chen, W. Chen, Z. Li, Y. Chi, C. Han, and S. Li, "Joint channel estimation and data rate maximization for intelligent reflecting surface assisted terahertz MIMO communication systems," IEEE Access, vol. 8, pp. 99 565-99 581, 2020.

[102] Y. Wu, F. Lemic, C. Han, and Z. Chen, "Sensing Integrated DFTSpread OFDM Waveform and Deep Learning-powered Receiver Design for Terahertz Integrated Sensing and Communication Systems," arXiv preprint arXiv:2109.14918, 2021.
[103] T. Mao, J. Chen, Q. Wang, C. Han, Z. Wang, and G. K. Karagiannidis, "Waveform Design for Joint Sensing and Communications in the Terahertz Band," arXiv preprint arXiv:2106.01549, 2021.

[104] L. T. Wedage, B. Butler, S. Balasubramaniam, Y. Koucheryavy, and J. M. Jornet, "Climate Change Sensing through Terahertz Communications: A Disruptive Application of 6G Networks," arXiv preprint arXiv:2110.03074, 2021.

[105] X. Zhang, C. Han, and X. Wang, "Joint beamforming-power-bandwidth allocation in terahertz NOMA networks," in 2019 16th Annual IEEE International Conference on Sensing, Communication, and Networking (SECON). IEEE, 2019, pp. 1-9.

[106] P. S. Bithas, E. T. Michailidis, N. Nomikos, D. Vouyioukas, and A. G. Kanatas, "A survey on machine-learning techniques for UAV-based communications," Sensors, vol. 19, no. 23, p. 5170, 2019.

[107] Y. Mekonnen, S. Namuduri, L. Burton, A. Sarwat, and S. Bhansali, "Machine learning techniques in wireless sensor network based precision agriculture," Journal of the Electrochemical Society, vol. 167, no. 3, p. 037522, 2019.

[108] X. Zhou, M. Sun, G. Y. Li, and B.-H. Fred Juang, "Intelligent wireless communications enabled by cognitive radio and machine learning," China Communications, vol. 15, no. 12, pp. 16-48, 2018.

[109] S. Helal, H. Sarieddeen, H. Dahrouj, T. Y. Al-Naffouri, and M. S. Alouini, "Signal Processing and Machine Learning Techniques for Terahertz Sensing: An Overview," arXiv preprint arXiv:2104.06309, 2021.

[110] W. Yuan, Z. Wei, S. Li, J. Yuan, and D. W. K. Ng, "Integrated sensing and communication-assisted orthogonal time frequency space transmission in vehicular networks," arXiv preprint arXiv:2105.03125v2, 2021.

[111] C. Huang, S. Hu, G. C. Alexandropoulos, A. Zappone, C. Yuen, R. Zhang, M. D. Renzo, and M. Debbah, "Holographic MIMO surfaces for $6 \mathrm{G}$ wireless networks: Opportunities, challenges, and trends," IEEE Wireless Communications, vol. 27, no. 5, pp. 118-125, 2020.

[112] N. Shlezinger, G. C. Alexandropoulos, M. F. Imani, Y. C. Eldar, and D. R. Smith, "Dynamic metasurface antennas for $6 \mathrm{~g}$ extreme massive mimo communications," IEEE Wireless Communications, vol. 28, no. 2 , pp. $106-113,2021$

[113] M. Nemati, S. R. Pokhrel, and J. Choi, "Modelling data aided sensing with UAVs for efficient data collection," IEEE Wireless Communications Letters, vol. 10, no. 9, pp. 1959-1963, 2021.

[114] Y. Gao, L. Liu, X. Zheng, C. Zhang, and H. Ma, "Federated sensing: Edge-cloud elastic collaborative learning for intelligent sensing," IEEE Internet of Things Journal, vol. 8, no. 14, pp. 11 100-11 111, 2021. 